\title{
Molecular and cellular heterogeneity of gastric cancer explained by methylation-driven key regulators
}

Seungyeul Yoo ${ }^{1,2,3}$, Quan Chen ${ }^{1,2,3}$, Li Wang ${ }^{1,2,3}$, Wenhui Wang ${ }^{1,2}$, Ankur Chakravarthy ${ }^{4}$, Rita Busuttil ${ }^{5}$, Alex Boussioutas, ${ }^{5}$, Dan Liu ${ }^{6}$, Junjun She ${ }^{6}$, Tim R. Fenton ${ }^{7}$, Jiangwen Zhang $^{8}$, Xiaodan Fan ${ }^{9}$, Suet-Yi Leung ${ }^{10}$, Jun Zhu ${ }^{1,2,3^{*}}$

1. Icahn Institute for Data Science and Genomics Technology, Icahn School of Medicine at Mount Sinai, New York, NY, USA

2. Department of Genetics and Genomic Sciences, Icahn School of Medicine at Mount Sinai, New York, NY, USA

3. Sema4, a Mount Sinai venture, Stamford, CT, USA

4. Princess Margaret Cancer Centre, University of Toronto, Ontario, Canada

5. Department of Medicine, University of Melbourne, Parkville, Victoria, Australia

6. Department of Surgery, First Affiliate Hospital, Xi' an Jiaotong University, Xi'an, China

7. School of Biosciences, University of Kent, Canterbury, UK

8. School of Biological Sciences, University of Hong Kong, Hong Kong, China

9. Department of Statistics, Chinese University of Hong Kong, Hong Kong, China

10. Department of Pathology, University of Hong Kong, Hong Kong, China

* Correspondence: jun.zhu@mssm.edu 


\begin{abstract}
Gastric cancer (GC) is a heterogeneous disease of diverse genetic, genomic, and epigenetic alterations. Tumor microenvironment (TME) also contributes to the heterogeneity of GC. To investigate GC heterogeneity, we developed an Integrative Sequential Causality Test (ISCT) to identify key regulators of GC by integrating DNA methylation, copy number variation, and transcriptomic data. Applying ISCT to three GC cohorts containing methylation, CNV and transcriptomic data, 11 common methylation-driven key regulators (ADHFE1, CDO1, CRYAB, FSTL1, GPT, PKP3, PTPRCAP, RAB25, RHOH, SFN, and SORD) were identified. Based on these 11 genes, gastric tumors were clustered into 3 clusters which were associated with known molecular subtypes, Lauren classification, tumor stage, and patient survival, suggesting significance of the methylation-driven key regulators in molecular and histological heterogeneity of GC. We further showed that chemotherapy benefit was different in the $3 \mathrm{GC}$ clusters and varied depending on the tumor stage. Both immune/stromal proportions in TME and tumor cell genomic variations contributed to expression variations of the 11 methylation-driven key regulators and to the GC heterogeneity.
\end{abstract}




\section{Introduction}

Gastric cancer (GC) is the fifth most common ( $8.2 \%$ of the total cancer cases) type of cancer and one of leading causes of global cancer death ${ }^{1}$. It occurs predominantly in Eastern Asian countries such as China, South Korea, and Japan ${ }^{2}$. In the United States, there were about 28,000 newly diagnosed GC patients and more than 10,000 deaths due to GC in $2017^{3}$. Many factors contribute to the high mortality and morbidity of GC. First, no distinct signs of symptoms of the disease until advanced stages and, hence, it is difficult to diagnose at early stage without routine screening via endoscopy ${ }^{4}$. Second, GC is heterogeneous in terms of biology, histology, and survival, and the diversity and complexity of the disease limits clear understanding of molecular mechanisms underlying in tumorigenesis, tumor progression, and metastasis of GC. Several studies have focused on the heterogeneity of GC in histology and/or in molecular characteristics. Lauren classification divides gastric tumors into three types (intestinal, diffuse or mixedindeterminate) based on histology ${ }^{5}$. The diffuse type tumors are associated with poorer prognosis and develop at earlier age, while the intestinal gastric tumors are associated with intestinal metaplasia and advanced age $\mathrm{e}^{6,7}$. Recent studies identified distinct molecular alterations by gene expression, genetics, epigenetics, and proteomics in $\mathrm{GC}^{8-11}$. The Cancer Genome Atlas (TCGA) GC study categorizes GC into four subtypes 1) Epstein-Barr virus (EBV) with positive infection by the virus, 2) Microsatellite instability (MSI), 3) Chromosomal instability (CIN), and 4) Genome stable (GS) tumors based on molecular features such as viral infection, DNA methylation, genome stability, and mutation burden ${ }^{10}$.

DNA methylation alteration is one of key factors contributing to GC heterogeneity. EBV tumors exhibit excessive increases of DNA methylation in genome-wide scale, CpG island methylator phenotype (CIMP) $)^{10,12}$. MSI GCs also show CIMP pattern while the level of hypermethylation is lower than tumors in EBV group ${ }^{10,13}$. GC clusters based on DNA methylation alterations such as CIMP were associated with clinical outcomes ${ }^{9}$. Also, it has been shown that 
genes involved in cancer-related pathways are more frequently affected by DNA methylations than by genetic alterations ${ }^{14}$. While these studies suggest a significant role of DNA methylation in gastric tumorigenesis and progression, detailed molecular mechanisms altered by DNA methylation changes are not well understood.

Copy number variation (CNV), a form of genomic alteration including amplification, or deletion of one or more sections of a chromosome, has a significant role in GC tumorigenesis ${ }^{15,16}$. Previous studies ${ }^{8,17,18}$ identify multiple CNV regions in GC, including gains of 3p22, 4q25, 8q24, $11 \mathrm{p} 13$, and $20 \mathrm{q} 13$ and losses of $1 \mathrm{p} 36$ and 9p21. It has also been shown that the number of CNV occurrences is higher in patients with metastasis than in patients without metastasis ${ }^{17}$. Deng et al. ${ }^{18}$ identify 13 genomic amplifications and 9 deletions containing multiple cancer related genes such as MYC, KRAS, CDK6 or $C D K N 2 A / B$. Wang et al ${ }^{8}$ integrate multi-omics data and identify consistent chromosomal changes in previous studies including gains on chromosomes 1q, 5p, 7, 9, 12 and 20 and losses on 1p, 3p, 4, 5q, 9p, 17p, 18q, 19p, 21, and 22. While an increasing number of $\mathrm{CNV}$ regions have been identified in $\mathrm{GC}$, it is still not fully understood how the associated genes impact tumorigenesis and progression of $\mathrm{GC}^{19,20}$.

The histological, molecular, and genomic heterogeneity is further convoluted with heterogeneity in the tumor microenvironment $(\mathrm{TME})^{21,22}$. Cancer, immune, and stromal cells have distinct transcriptomic, genomic, and epigenetic patterns. In bulk tissue transcriptomic data, gene expression correlations may be due to TME heterogeneity or genomic heterogeneity (colocalized in deletion or amplification blocks) instead of transcriptional co-regulations, which hinder our understanding biology of GC. Also, the heterogeneity in TME is associated with prognosis or drug response. For example, higher proportion of immune cells in the tumor microenvironment is associated with better survival ${ }^{23-25}$ and better response to checkpoint blockade immunotherapies ${ }^{26-28}$. On the other hand, a higher proportion of stromal cells is associated with worse survival ${ }^{29}$, especially in $\mathrm{GC}^{30-32}$, and worse response to checkpoint 
blockade immunotherapies $^{33}$. However, molecular connections of TME with different GC heterogeneities have not been systematically examined.

Here we describe an integrative causal model for identifying potential regulatory mechanisms in GC and their relationship with GC heterogeneity by integrating multi-omics data including gene expression, DNA methylation, and CNV profiles. Previously, we modeled a causality test as an empirical Bayesian estimation of the significance of cis DNA methylation regulated genes affecting other genes (trans) ${ }^{34}$. The previous causal model considered only one factor in gene expression regulation while there are multiple elements in the complex transcriptional regulation mechanisms. Therefore, we propose a new model here, Integrative Sequential Causality Test (ISCT), simultaneously considering transcriptional regulations driven by CNVs and methylation variations. We use the cis-association with promoter methylation and/or copy number alterations as priors and test whether the trans-associations are mediated through expression of cis-genes. (Figure 1A). Then, key regulators are inferred based on the number of downstream genes (Figure 1B). We applied ISCT to three GC cohorts (Supplementary Table 1) in which DNA methylation, CNV, and gene expression data were available, and highlighted key regulators and their downstream genes (Figure 1C). Eleven methylation-driven key regulators (ADHFE1, CDO1, CRYAB, FSTL1, GPT, PKP3, PTPRCAP, RAB25, RHOH, SFN, and $S O R D$ ) were identified in all three datasets. Based on the common methylation-driven key regulators, GC samples were divided into three groups, each with distinct clinical outcome. The findings were validated in five independent GC cohorts (Supplementary Table 1). Among the 11 common methylation-driven key regulators, FSTL1 expression was significantly associated with survival in 5 out of 7 datasets in which survival information were available. In GC cell lines, FSTL1 was regulated epigenetically, and its correlated genes in cell lines were significantly enriched for its downstream genes inferred in bulk tissue profiles, consistent with a potential key regulator role of FSTL1 in cell lines. To dissect the sources of gene expression variations of the 11 common methylation-driven key regulators, cellular compositions of bulk tissues were 

available under aCC-BY-NC-ND 4.0 International license.

computationally inferred and their relationships with key regulators were investigated in the context of heterogeneity of gastric cancer (Figure 1C). FSTL1 expression was highly correlated with the stromal cell fraction, suggesting both tumor intrinsic signals and variations in stromal cells in TME could regulate tumor cells. 


\section{Results}

\section{Comparison of ISCT and mediation tests on simulated datasets}

Integrative Sequential Causality Test (ISCT) is developed for simultaneously identifying transcriptional regulations driven by CNVs and methylation variations. The ISCT approach and mediation tests ${ }^{35,36}$ share some similarities in identifying relationships of gene expression $g$ causally regulated by DNA methylation $m$ and $\mathrm{CNV} c$ (Figure 1A). ISCT assumes sequential events occur in biology (Methods) while mediation tests take DNA methylation and CNV data as covariates without making any assumption. To systematically compare the performance of each method in identifying causal transcriptional regulations, we simulated two datasets, one based on a causal/mediation model and one based on an independent model according to the correlation distributions of the HKU dataset (detailed in Methods). We applied ISCT and two mediation tests to detect the underlying causal/mediation relationships. For the mediation tests, we conducted Baron and Kenny's mediation test ${ }^{36}$ (referred to as "mediation test" in the following text and figures) to test for complete mediation, corresponding to the causal path tested in the ISCT approach; we also conducted the Sobel test ${ }^{37}$ to assess the significance of mediation effect, which does not necessarily guarantee complete mediation. In the dataset simulated based on a causal model (simulation \#1), ISCT identified $91.7 \%$ of pairs as significant while the mediation test and the Sobel test identified 39.2\% and $23.7 \%$ as significant, respectively (Figure 2A). In the dataset simulated based an independent model (simulation \#2), the false positive rate of ISCT estimated by the proportion of simulated independent pairs tested significant was $0.46 \%$ while the false positive rates of the mediation test and the Sobel test were $0.40 \%$ and $0.31 \%$, respectively (Supplementary Figure 1A). Therefore, the ISCT method demonstrated significantly higher power in detecting causal pairs comparing with other mediation tests while the false positive rate was well-controled under $0.5 \%$ similar to the other mediation tests. 
To systematically investigate the scenarios where these methods differ, we conducted further simulation studies given certain correlation levels among the variables to investigate the performance of each method under each specific scenario. We performed two additional simulations (simulations \#3 and \#4). In simulation \#3, we randomly selected 10,000 cis-trans pairs from the HKU cohort with significant cis- and trans-relationships (detailed in Methods). This data-generating process mimiced the effect of a mediator ${ }^{38}$, by leaving the correlation between the dependent and the independent variables to vary. The power to identify causal relationships increased as the strength of the correlation between the dependent and the independent variables increased (Figure 2B). The ISCT method consistently outperformed the mediation test and the Sobel test with higher detection power at all correlation levels. At the correlations between the dependent and the independent variables range 0.3 0.6 (Figure 2B), the Sobel test had the lowest detection power; the mediation test had minimal power at the correlation level of 0.3 , around $40 \%$ power at the correlation level of 0.4 , and reached over $85 \%$ power at the correlation level of 0.5 ; while ISCT had $>75 \%$ power at the correlation level of 0.3 , around $90 \%$ power at the correlation level of 0.4 , and reached over $95 \%$ power at the correlation level of 0.5 (Figure 2B). These results suggested that the ISCT method was better powered in detecting causal pairs at lower correlation levels, where most pairs in the HKU cohort were observed (Figure 2C).

To investigate the performance of the two methods in the presence of colinearity, we simulated a fourth dataset (simulation \#4, detailed in Methods). At the correlation between the dependent variable and the mediator around 0.5 , the power of both mediation methods dropped with the increase in colinearity, and the drop in power with colinearity was more drastic when the correlation was around 0.4 0.5 (Figure 2D). In contrast, the power of ISCT did not decrease with the increase in colinearity (Figure 2D). The results from simulations suggest that ISCT considering sequencial biological events in the model outperform typical mediation methods with higher detection power in the presence of colinearity. 


\section{Methylation-driven key regulators}

Both methylation and CNVs play a significant role in GC tumorigenesis and progression and should be simultaneously considered in transcriptional regulation as modeled in ISCT (Figure 1A).

1) cis regulation driven by DNA methylation

We first investigated cis transcriptional regulations of $g_{x}$ by DNA methylation in their promoter regions $m_{x}$ and CNV alteration $c_{x}$ based on a linear regression model, $g_{x} \sim m_{x}+c_{x}$ for gene $x$ (detailed in Methods). At FDR<0.05, 7,917, 46,459, and 1,536 cis-methylation probes whose methylation levels were negatively correlated with the corresponding gene expression levels, which were summarized to $2,915,11,880$, and 1,119 cis-methylation genes, in the HKU, TCGA, and Singapore datasets, respectively. Among them, 474 cis-methylation genes were common among all three datasets (Figure 3A), which were enriched for signatures of ESTROGEN_RESPONSE_LATE and ESTROGEN_RESPONSE_EARLY in Hallmark gene sets from MSigDB ( $\mathrm{p}=1.1 \times 10^{-11}$ and $2.3 \times 10^{-5}$, respectively). It is known that there is a higher GC risk in men than in women ${ }^{39}$ and estrogen plays an important role in GC tumorigenesis and $\operatorname{progression}^{40}$. We tested whether the methylation levels of the cis-methylation genes enriched for estrogen signatures were associated with the sex of patients, but this was not the case (Supplementary Figures 2A\&B). In addition, most of them (17 and 24 out of 33 genes in HKU and Singapore dataset, respectively) were differentially methylated (t-test FDR $<0.01$ ) between tumor and adjacent normal tissues (Supplementary Figure 2C). Our result suggests that the estrogen response pathway is regulated at the epigenetic level.

2) trans regulations associated with DNA methylation

For each cis-methylation probe, we collected trans-methylation genes whose expression levels significantly correlated with the methylation variations (detailed in Methods). At the FDR $<0.05$, 695,696, 70,636,038, and 870,242 trans-methylation genes were identified in the HKU, TCGA, 
and Singapore datasets, respectively. The trans-methylation regulations significantly overlapped among different datasets (Figure 3B). Based on background of commonly significant cismethylation genes among the three dataset, $70 \%$ and $66 \%$ of trans regulated gene pairs in HKU and Singapore were shared with the ones identified in the TCGA (FET Fold Enrichments (FEs)= 2.3 and 2.1 with $\mathrm{p}<1.0 \times 10^{-256}$ for HKU and Singapore, respectively). While with smaller percentage (about 5\% and 18\%), the trans-methylation gene pairs based on the HKU and Singapore dataset, respectively, were also significantly shared (FET FE $=4.3$ and $\mathrm{p}<1.0 \times 10^{-256}$ ). Moreover, for the common trans-methylation gene pairs among different cohorts, the directions of the trans association were consistent (Figure 3C) suggesting similar impacts of methylation variations on the trans genes.

3) Causal relationships driven by DNA methylation variations

After identifying cis- and trans- methylation genes, we further tested putative causal relationships between cis- and trans-methylation genes by testing whether a cis-methylation gene and a transmethylation gene were independent given the expression of cis-genes (Methods). With the independence test $p$-value cutoff $>0.01,102,522,19,085,499$, and 353,576 causal gene pairs were identified in the HKU, TCGA and Singapore datasets, respectively (Supplementary Figure 3).

4) Comparison of causal pairs based on ISCT and mediation tests

We took the HKU cohort as an example to investigate the difference between the causal relationships detected by ISCT and mediation test. Out of the 994,896 candidate cis-trans probe pairs, 143,332 pairs (summarized to 102,522 gene pairs) were identified as causal by ISCT while 53,267 pairs were identified as causal relationships with full mediation by Baron and Kenny's mediation test. The majority of the causal pairs identified by Baron and Kenny's mediation test were identified by the ISCT approach as well (Supplementary Figure 1B). To further investigate the scenarios where the two methods differ, we examined the distribution of gene expression correlations for the cis-trans gene pairs detected by each method. Both methods require a significant correlation between $g_{x}$ and $g_{y}^{*}$ to detect a causal relationship, whereas the ISCT 
method was able to detect more pairs with significant causal relationships at a lower correlation level (Figure 2). In addition, the causal pairs detected by ISCT contained a higher faction of pairs with strong anti-correlations between $g_{x}$ and $m_{x}$, suggesting a possible power increase in the presence of colinearity.

5) Methylation-driven key regulators

We next assessed whether there were any regulators whose methylation variations casually regulated expression levels of many other genes in trans (downstream genes), which were defined as methylation-driven key regulators (Methods). The number of downstream genes causally associated with each of cis-methylation genes followed a scale-free distribution (Supplementary Figure 4); most cis-methylation genes regulated a small number of downstream genes while a few cis-methylation genes regulated a large number of downstream genes. At the cutoffs based on reflection points (detailed in Methods, Figure 3D), 221, 2,049, and 226 methylation-driven key regulators were identified in the HKU, TCGA, and Singapore datasets, respectively. Among them, 232 were identified in at least two datasets and 11 were identified in all three datasets (Figure 3E and Supplementary Table 2). The 11 common methylation-driven key regulators were located on multiple chromosomes, chr1 (RAB25 and SFN), chr3 (FSTL1), chr4 (RHOH), chr5 (CDO1), chr8 (ADHFE1 and GPT), chr11 (CRYAB, PKP3, and PTPRCAP), and chr15 (SORD).

6) Downstream genes of the methylation-based key regulators

For each key regulator, its downstream genes were further split into two groups; ones positively or negatively correlated with expression of the regulators. The downstream genes were significantly overlapped among all three datasets with consistent direction of regulations (Supplementary Table 2) suggesting similar impacts of the key regulators. We further determined downstream genes of the 11 common methylation-driven key regulators commonly identified at least in two datasets (Supplementary Table 3). Interestingly, the 11 methylation-driven key regulators shared similar set of their downstream genes (Figure EV1A). We performed functional enrichment test of the downstream genes of the methylation-driven key regulators against 
signature sets in MSigDB databases ${ }^{41,42}$. The downstream genes of multiple methylation-driven key regulators were commonly enriched for three groups of Hallmark gene sets (Figure EV1B). One group of downstream genes, which were positively regulated by FSTL1, CRYAB, CDO1, and ADHFE1 and negatively regulated by PKP3, SORD, GPT, RAB25, and SFN expression, was enriched for genes involved in EMT, MYOGENESIS (FET p-values $=2.3 \times 10^{-15}$ and $5.5 \times 10^{-14}$, respectively for positively regulated downstream genes of FSTL1) while their anti-correlated downstream genes were enriched for cell cycle related functions such as E2F_TARGETS, G2M_CHECKPOINT, and MITOTIC_SPINDLE (FET p-values $=4.8 \times 10^{-24}, 9.7 \times 10^{-14}$ and $5.6 \times 10^{-}$

${ }^{6}$, respectively for negatively regulated downstream genes of FSTL1). The downstream genes positively regulated by gene expression of $R H O H$ and PTPRCAP were enriched for immune related functions such as INFLAMMATORY_RESPONSE, ALLOGRAFT_REJECTION, and INTERFERON_GAMMA_RESPONSE (for positively regulated downstream genes of $R H O H$ pvalues $=5.2 \times 10^{-18}, 3.2 \times 10^{-37}$, and $1.5 \times 10^{-16}$, respectively).

\section{GC subtypes based on methylation-driven key regulators}

Methylation-driven key regulators were correlated in GC

Based on gene expression similarity, the 11 common methylation-driven key regulators were clustered into two anti-correlated groups with CDO1, CRYAB, FSTL1, ADHFE1, RHOH, and PTPRCAP in one group and GPT, SORD, PKP3, RAB25 and $S F N$ in the other (Figure 4A). The first group was further separated into two gene clusters in which CDO1, CRYAB, FSTL1, and ADHFE1 (G1) were co-expressed while RHOH and PTPRCAP (G2) were closely expressed. Five genes in the other group, GPT, $S O R D, P K P 3, R A B 25$, and $S F N(\mathrm{G} 3)$, were also highly correlated with one another. These patterns were consistently observed across all GC cohorts in the study (Figure 4A).

Tumor clusters based on the gene expression of methylation-driven key regulators 
Tumor samples were clustered by k-means clustering into three groups based on the similarity of the expression of the 11 common methylation-driven key regulators (Figure 4B). The three groups, $\mathrm{C} 1$ to $\mathrm{C} 3$, showed increasing expression levels of genes in G1 and G2, while decreasing expression of genes in G3 and the samples in all GC datasets were clustered in a similar manner (Figure 4B). Samples in the groups C1 and C3 showed opposite expression patterns for the genes in G1 and G3. C2 samples showed intermediate expression of genes in G1 and G3 (Figure 4B).

Then, we investigated whether the tumor clusters based on key methyl regulator expression levels were associated with previously known molecular features of GC. The tumor clusters were significantly overlapped with Lauren classifications (Figure 4C and Supplementary Table 4). The $\mathrm{C} 1$ cluster was enriched for the intestinal subtype in all datasets except for CPTAC dataset in which most of samples are the diffuse subtype (FET FEs= 2.77, 1.5, 2.07, 1.97, and 1.99 and p-values $=4.5 \times 10^{-8}, 1.1 \times 10^{-5}, 0.0003,2.8 \times 10^{-7}$, and 0.005 in HKU, TCGA, Singapore ACRG, and Australia datasets, respectively). On the other hands, the C3 group was enriched for diffuse subtype $\left(\right.$ FET FEs $=1.75,6.01,3.14,1.93$, and 2.99 and $p$-values $=0.05,6.0 \times 10^{-17}, 4.9 \times 10^{-}$ ${ }^{5}, 3.3 \times 10^{-5}$, and $3.9 \times 10^{-5}$ in HKU, TCGA, Singapore, ACRG, and Australia datasets, respectively).

In addition, tumors in $\mathrm{C} 1$ significantly overlapped with the MSI and CIN subtype in TCGA (FET FEs=2.17 and 1.3, p-values=0.003 and 0.02, respectively), the proliferative subtype in Singapore and Australia datasets (FET FEs $=7.74$ and 4.69 , p-values $=2.8 \times 10^{-10}$ and $1.4 \times 10^{-7}$, respectively), and also the MSS/TP53- subtype in ACRG (FET FE=1.83, p-value=0.009), and Immunogenic subtypes in CPTAC dataset (FET FE=2.11 with p-value=0.04). Tumors in C2 significantly overlapped with the metabolic subtype in Singapore and Australia datasets (FET $\mathrm{FEs}=2.58,12.19, \mathrm{p}$-values $=0.03,4.5 \times 10^{-6}$, respectively). Tumors in the $\mathrm{C} 3$ were enriched for GS subtype in the TCGA dataset $\left(\right.$ FET FE $=5.37$, p-value $=8.6 \times 10^{-12}$ ). Interestingly, the $\mathrm{C} 3$ cluster was also highly enriched for mesenchymal-like subtype tumors in Singapore, ACRG, and Australia $\left(\right.$ FET FEs $=18.08,27.5$, and 9.6, p-values $=3.9 \times 10^{-8}, 7.8 \times 10^{-27}$ and $2.4 \times 10^{-13}$, respectively $)$. While a previous study reported the TCGA GS subtype was not equivalent to the ACRG MSS/EMT 
subtype $^{43,44}$, our results suggest that they shared similar molecular features characterized by the 11 methylation-driven key regulators.

The C1-3 clusters were also associated with tumor stages in TCGA, Singapore, ACRG, and Yonsei datasets (Figure 4C and Supplementary Table 4). The results were consistent with previous reports that mesenchymal-like GC tumors were associated with diffuse type and more advanced tumors while the proliferative subtype was associated with intestinal type $\mathrm{e}^{10,43,45}$. Taken all together, these results further suggest significant roles of the methylation-driven key regulators in the heterogeneity of GC tumors.

\section{Survival differences among the tumor clusters}

The clusters based on the methylation-driven key regulators showed prognostic differences (Figure 4D). In general, patients in the $\mathrm{C} 1$ group had better survival than ones in the $\mathrm{C} 3$ and the C2 group had intermediate survival, whether more closely to $\mathrm{C} 1$ or $\mathrm{C} 3$ depending on their similarity of expression patterns of the methylation-driven key regulators (Figure 4B). The survival differences between tumors in the $\mathrm{C} 1$ and $\mathrm{C} 3$ were statistically significant in TCGA, Singapore, ACRG, and Yonsei datasets (Likelihood Ratio (LR) test p-values=0.04, 0.03, 2.3×10 ${ }^{-7}$, and 0.0008, respectively). In HKU and SMC datasets, the C1 and C3 clusters showed different survival compared to each other, while LR test p-values were not significant. The clusters were not associated with patients' survival in the Australia datasets. It was worthy to note that the patients in Australia dataset were mixed with or without 5-FU treatment which showed significant survival differences within the same subtypes ${ }^{45}$. Combining all results above, the methylation-driven key regulators classify samples into three groups, which well agreed with molecular and histological heterogeneity as well as differences in clinical outcomes of gastric cancer.

Chemotherapy response differences among tumor clusters 
A recent study reported by Oh et al. showed distinct genomic features between epithelial and mesenchymal phenotype (EP and MP) of gastric tumors ${ }^{46}$. They used a set of 299 signature genes, which included FSTL1 and GPT, to classify gastric tumors into two groups. When comparing the tumor clusters based on the 11 methylation-driven key regulators with their EP/MP results using overlapping samples in the TCGA and ACRG datasets, C1 and C3 almost exclusively overlapped with EP and MP, respectively (Figures 5A, details in Appendix, Supplementary Table 5). This is consistent with the result that the positively regulated downstream genes of FSTL1 were enriched for EMT pathways (FET p-values $=2.3 \times 10^{-15}, \mathrm{EV} 1 \mathrm{~B}$ ). We analyzed the 4 additional GC datasets used in Oh et al study (Methods), and tumor clusters based on the 11 methylation-driven key regulators showed similar associations with EP and MP groups (Figures 5B\&C, Supplementary Table 5). These results are consistent with our previous observation that $\mathrm{C} 3$ tumors were significantly associated with invasive or mesenchymal subtype of tumors in the Singapore and Australia datasets (Figure 4C and Supplementary Table 4). The tumors in C1 and C3 groups showed significant differences in overall survival as well as recurrence free survival (Figures 5D\&E) consistently with distinct survival patterns between EP and MP groups ${ }^{46}$. These results suggest that the epithelial/mesenchymal phenotype can be largely explained by our methylationdriven key regulators.

Oh et al reported that adjuvant chemotherapy (CTX) was effective exclusively for EP tumors $^{46}$. Following their procedure, we combined patients from KUGH, YUSH, and KUCM datasets, observed differences in the recurrence free survival between patients with and without CTX were significant only for $\mathrm{C} 1$ group $(\mathrm{p}=0.039)$ but not for $\mathrm{C} 2$ and $\mathrm{C} 3$ groups $(\mathrm{p}=0.44$ and 0.12, respectively, Figure 6A), and similarly significantly in the EP cluster not in MP cluster ( $p=0.009$ and 0.98 , respectively, Figure 6A) as reported by Oh et al.

However, in each tumor cluster (C1-3 clusters by our method or EP/MP cluster by Oh et al), there was a mixture of tumors at different stages with poor survival for patients of advanced stage tumors (Figure 6B). When examining tumors according to molecular clusters and stages, 
patients benefited from CTX only when tumors were in the C1 cluster and at Stage III ( $\mathrm{p}=0.006)$ based on our clustering method or in the EP cluster and at Stage III $(\mathrm{p}=0.004)$ based on Oh et al (Figures 6C-E). There might be CTX treatment benefit for tumors in the C3 cluster and at Stage II $(\mathrm{p}=0.11)$ or at stage III $(\mathrm{p}=0.08)$. These results suggest strong needs for personalizing treatment decision based on molecular phonotype as well as tumor stage.

\section{Clinical significance of the methylation-driven key regulators}

Next, we associated the expression levels of the methylation-driven key regulators in tumors with patients' survival and tumor stages in the 7 datasets ( 3 integrative datasets and 4 gene expression validation datasets except for CPTAC) and strong associations between them were observed with consistent directions among multiple datasets (Figures 7A\&B, Supplementary Table 6). The increased expressions of the $\mathrm{G} 1$ genes (CDO1, CRYAB, FSTL1, and ADHFE1) were significantly associated with poor prognosis as well as advanced tumor stages in multiple datasets. A recent study showed that $C R Y A B$ overexpression induced invasion and migration via EMT in gastric cancer cells $^{47}$. FSTL1 expression showed the most consistent and strongest association with both survival and tumor stages within 5 datasets (survival: p-values $=0.0002,1.1 \times 10^{-6}, 0.02,0.0004$, and 0.04 for Singapore, ACRG, Australia, Yonsei and SMC datasets, respectively; tumor stages: $\mathrm{p}$-values $=0.0003,0.01,6.9 \times 10^{-5}, 0.002$, and $9.3 \times 10^{-5}$ for HKU, TCGA, Singapore, ACRG, and Yonsei datasets, respectively). FSTL1 was reported as a key mediator in immune dysfunction driven by metastasis and aging in mouse cancer models ${ }^{48}$ but no functions of it in gastric cancer was reported previously.

On the other hand, the expression levels of the G3 genes (GPT, SORD, PKP3, RAB25, and $S F N$ ) were associated with good prognosis and early stages (Figures 7A\&B, Supplementary Table 6). It has been shown that loss of PKP3 protein expression indicated an invasive phonotype by comparing immunohistochemistry of PKPs in gastric tumor and normal gastric 
tissues $^{49}$. Functional roles of other tumor suppressor like genes such as GPT, SORD, and SFN in GC have not been addressed before.

We further compared the downstream genes of the methylation driven key regulators (Supplementary Table 3) with gastric cancer survival defined based on ACRG dataset and progression signature based on early and advanced GC profiles in the Vecchi et al. study ${ }^{50}$ (Methods). These signatures were further split into "good" or "poor" according to the direction of their associations with patient survival (Supplementary Table 7). Most of the methylation-driven key regulators, except for $R H O H$ and PTPRCAP, were survival-associated genes and their downstream genes were also significantly overlapped with survival signatures (FET p-value $<10^{-}$ ${ }^{20}$, Figure 7C). The downstream genes were also consistently associated with gastric cancer progression signatures (Figure 7C). It is worth to note that downstream genes of FSTL1 showed the strongest association with both survival and progress signatures among all methylation-driven key regulators, which was consistent with our observations that FSTL1 expression was significantly associated with poor survival and tumor stages in multiple GC datasets (Figures 7A\&B, Supplementary Table 6).

\section{Tumor intrinsic variations of the methylation-driven key regulators in cancer cells}

Tumor-stroma interactions in GC are associated with prognosis ${ }^{30-32}$ and contribute to molecular heterogeneity ${ }^{21}$. Hence, we investigated whether variations of the methylation-driven key regulators were from tumor cells or associated with TME. First, we checked the methylation and expression levels of the 11 methylation-driven key regulators in CCLE gastric cancer cell lines ${ }^{51}$, 52. Based on gene expression of CCLE cancer cell lines, FSTL1 was the only gene in the G1 group having largely varying expression among 36 cell lines while others such as $C D O 1, C R Y A B$, and ADHFE1 were not highly expressed (Figure 8A). Interestingly, FSTL1 expression increased in tumor tissues compared to non-tumor gastric tissues consistently in TCGA and HKU datasets while the other three showed an opposite pattern (Figure EV2). Considering their expressions had 
highly correlated each other (Figure 4A), FSTL1 expression might be derived more from tumor cell variations than $C D O 1, C R Y A B$, and $A D H F E 1$. Especially, $C D O 1$ was low expressed in cancer cells $(\log 2(\mathrm{RSEM})<1$ in 34 out 36 cell lines) suggesting pro-tumor progression property of CDO1 might be not from tumor cells but other factors. Among the genes in the G3 group (GPT, SORD, $P K P 3, R A B 25$, and $S F N$ ), which tumor-suppressor like properties were observed in, GPT was less expressed than others (Figure 8A) as well as down-regulated expression in tumor tissues from non-tumor gastric tissues (Figure EV2). These results suggest that the source of expression variations of the methylation-driven key regulators was not the same even though their expressions were highly correlated in bulk tissues.

The CCLE methylation data within $1 \mathrm{~kb}$ from TSS for the methylation-driven key regulators showed opposite patterns from the gene expression (Figure 8B). Four methylationdriven key regulators (ADHFE1, FSTL1, GPT, and SFN) were anti-correlated between the methylation level and gene expression (Figure 8C) suggesting that these genes were suppressed by promoter methylation in gastric cancer cells.

Furthermore, to test whether the transcription of downstream genes was driven by expression variations of the methylation-driven key regulators in gastric cancer cells, we compared the gene expression of the methylation-driven key regulators and their downstream genes in GC cell lines in two different ways. First, we identified significantly correlated genes with the 4 methylation-driven key regulators (FSTL1, ADHFE1, GPT, and SFN), which were cisregulated by their promoter methylation level in GC cell lines (Figure 8C), then compared the downstream genes based on bulk tissue data and correlated genes based on cell line data. The downstream genes of the all of the 4 methylation-driven key regulators significantly enriched for genes significantly correlated with the corresponding key regulators in the CCLE GC cell lines and also the directions of the associations were consistent for all 4 genes (Figure 8D). Next, we also compared the distribution of the tumor intrinsic correlation between the methylation-driven key regulators with their downstream genes with that of background genes. The downstream 
genes showed clear different associations with their key regulators compared to background genes with consistent direction of correlation (Figure 8E). The cumulative distributions of the correlation coefficients were compared between the downstream genes and background genes via Kolmogorov-Smirnov (KS) Tests and all comparisons were statistical significant (KS-test D=0.27 and 0.39 with $\mathrm{p}$-values $=3.7 \times 10^{-30}$ and $7.4 \times 10^{-96}$ for positively and negatively regulated downstream genes of FSTL1, Supplementary Figure 5).

These results suggest that the expression of the methylation-driven key regulators was cis-regulated by their promoter region methylation and the putative causal relationships with their downstream genes were partially driven by tumor intrinsic variations of the genes.

\section{Influence of TME on the methylation-driven key regulators}

Next, we examined cellular compositions of the bulk gastric tumors based on gene expression and DNA methylation (MethylCIBERSORT) ${ }^{53}$ (Methods). Cell proportions based on gene expression and DNA methylation profiles were similar (Pearson correlation $\mathrm{p}=2.3 \times 10^{-37}, 3.9 \times 10^{-58}$, and $6.4 \times 10^{-48}$ for immune, stromal, and cancer cells, respectively, Figure EV3). The expression levels of the 11 methylation-driven key regulators were significantly correlated with cell compositions (Supplementary Figure 6). The genes in the G1 group (CDO1, CRYAB, FSTL1, and ADHFE1) were highly associated with stromal proportions while ones in the G2 group (RHOH and PTPRCAP) were significantly correlated with immune proportions. The expression levels of the G3 genes (GPT, SORD, PKP3, RAB25, and $S F N)$ were anti-correlated with stroma and immune proportions.

Next, we investigated the distribution of cellular compositions in the tumor clusters. In general, the immune and stromal cell proportions increased from the $\mathrm{C} 1$ to the $\mathrm{C} 3$ clusters while cancer cell proportions decreased (Supplementary Figure 7). Furthermore, the cellular compositions were tested for their association with patients' survival (Supplementary Table 8).

As the stromal proportions were correlated with the expression of the methylation-driven key 
regulators in the G1 group, they were significantly associated with poor survival in multiple datasets $\left(\right.$ Hazard Ratios $(H R s)=1.41,1.62,1.35$ and 1.22, p-values $=0.008,5.4 \times 10^{-8}, 2.4 \times 10^{-6}$ and 0.02 for Singapore, ACRG, Yonsei and SMC, respectively) consistently with previous reports showing poor survival associated with higher tumor-stroma interactions ${ }^{30-32}$.

As the methylation-driven key regulators potentially regulated a large number of downstream genes in each dataset (Figure 3D) and the methylation-driven key regulators were highly correlated with cell type proportions, we also tested whether the downstream genes were influenced by cell type proportion as well. Indeed, downstream target genes showed stronger association with cell type proportions compared to other trans genes suggesting influence of TME in the causality test (Appendix and Supplementary Figure 8). The results based on the CCLE cancer cell lines as well as cell type proportions together suggest that the transcription regulations of the methylation-driven key regulators on their downstream genes were influenced by both TME and intrinsic variations of cancer cells.

Taken all results together, tumor cells and their interactions with TME may drive molecular and cellular heterogeneity of gastric cancer which, in turn, leads to heterogeneity of clinical outcomes of GC patients. 


\section{Discussions}

Gastric cancer is of highly heterogeneous molecular and histological features. These molecular and cellular heterogeneities were driven by complex and diverse genomic and epigenetic alterations occurred during tumorigenesis and progression of gastric cancer. We integrated DNA methylation, $\mathrm{CNV}$, and gene expression data to understand these heterogeneities, and developed the ISCT procedure to infer causal relationships between a pair of cis-methylation gene and trans genes. Among the numerous numbers of significantly associated cis-trans gene pairs, ISCT identified potential causal gene pairs whose significant associations were mediated by expression of cis genes that were driven by methylation variations. Based on simulation studies, we showed that ISCT outperformed mediation tests with consistently higher detection power and was robust against to colinearity problems. It is not trivial to systematically evaluate the performance of ISCT in empirical data, thus we focused only on coherent common observations from the three multi-omics GC datasets (HKU, TCGA, and Singapore). In addition, we collected gene expression data from multiple independent GC cohorts to cross-validate our observations.

By applying ISCT, we identified 11 methylation-driven key regulators common from all three datasets. These genes were further grouped into three groups based on their gene expression similarity (Figure 4A): G1 (CDO1, CRYAB, FSTL1, and ADHFE1), G2 (RHOH and PTPRCAP), and G3 (GPT, PKP3, RAB25, SFN, and SORD). The G1 genes showed strong association with poor survival while the expression of G3 was associated with good prognosis (Figure 7).

Based on expression of the 11 methylation-driven key regulators, GC samples were grouped into three clusters ( $\mathrm{C} 1$ to $\mathrm{C} 3)$ which showed distinct molecular or clinical features (Figure 4). Samples in C3 cluster were significantly enriched for diffuse type tumors in all datasets while samples in $\mathrm{C} 1$ were for intestinal type ones. In addition, C3 samples were enriched for the GS subtype defined in TCGA cohort. While MSI tumors were well known to have CIMP features $^{54}$, there was no previously reported association between methylation with GS tumors. 
The C3 samples were also enriched for mesenchymal-types (or invasive) features determined in Singapore, ACRG, Australia, and CPTAC cohorts. Interestingly, we observed correlation of the sample clusters and tumor stage, and samples in C3 showed enrichment for advanced stages and also worst survival outcomes in multiple cohorts (HKU, TCGA, Singapore, ACRG, Yonsei and SMC dataset, Figure 4D). Previous studies about DNA methylation in GC mostly focused on molecular subtypes defined based on CIMP status ${ }^{9,12,55}$ but the association of CIMP tumors with clinicopathological features were not well agreed in all studies. ISCT enables us to identify methylation-driven key regulators. The associations of the key methylation-driven regulators with molecular and clinical features were consistent across multiple independent GC cohorts (Supplementary Tables 4 and 6).

Comparing tumor clusters based on methylation-driven key regulators with reports by Oh et al., the $\mathrm{C} 1$ and $\mathrm{C} 3$ tumors were enriched for EP and MP, respectively. Moreover, we showed the adjuvant chemotherapy sensitivity in $\mathrm{C} 1-3$ or EP/MP clusters was stage dependent, suggesting significance of considering not only molecular features but also progression of tumors when treating GC patients.

Some of the methylation-driven key regulators were reported to play a significant role in GC. Chen et al. showed that overexpression of $C R Y A B$ induced EMT, migration, and invasion of gastric cancer cells in vitro and in vivo as well as reversing these phenomena by silencing $C R Y A B^{47}$. In addition, they showed strong associations between CRYAB expression and cancer metastasis and survival outcomes in patients. These results were consistent with our results suggesting $C R Y A B$ as a potential oncogene regulating EMT associated genes (Figure 7 and Figure EV1B). Demirag et al. investigated IHC of plakophilins in gastric adenocarcinoma and normal gastric tissues and reported low PKP3 protein levels were correlated to the node number, tumor stages, and poor prognosis in gastric carcinoma ${ }^{49}$, which was consistent with our result that $P K P 3$ was associated with good survival and down-regulated in advanced stages. ADHFE1 was reported as an oncogene by inducing metabolic reprogramming in breast cancer ${ }^{56}$, but no previous 
association with gastric cancer was reported. Interestingly, Oh et al. also observed upregulation of IGF1 coupled with promoter region hypomethylation in mesenchymal gastric tumors and showed inhibition of $I G F 1$ reduced tumor growth in mesenchymal type tumor cells ${ }^{46}$. IGF1 was identified as one of methylation-driven key regulators in the Singapore and TCGA dataset (Supplementary Table 2).

Contrary to those positive controls, the observation with Cysteine dioxygenase type 1 (CDO1) was not consistent with previous reports. Hao et al. showed that suppressing CDO1 increased ferroptosis resistance in human gastric cancer cells and tumors in CDO1 knockdown mice grew faster compared to controls ${ }^{57}$. Harada et al. reported hypermethylation of $C D O 1$ promoter as an independent prognostic marker in gastric cancer ${ }^{58}$. This discrepancy could be explained by very low expression in cancer cells (Figure 6A), downregulated expression in tumors compared to normal tissues (Figure EV2), and strong association of $C D O 1$ expression with stromal proportions (Supplementary Figure 6), together suggesting that the CDOI expression in bulk tumors was mainly determined by stroma cell proportion, which is an independent prognostic factor in $\mathrm{GC}^{30}$.

Other methylation-driven key regulators have not been previously associated with gastric cancer. Follistatin like 1 (FSTL1) was associated with tumor cell proliferation, migration, and invasion in several other cancers including lung, colon, breast and renal cell carcinoma ${ }^{59-62}$, but had not distinctively been linked with gastric cancer. Our results suggest FSTL1 as a potential novel oncogene in gastric cancer based on its over-expression in tumor samples and strong association with patients' survival and tumor stages in multiple GC datasets (Figure 7, Supplementary Table 6). While FSTL1 expression in the bulk was also strongly correlated with stromal proportions like CDO1 (Supplementary Figure 6), FSTL1 expression was associated with its promoter methylation in gastric cancer cells (Figure 8A-C). Moreover, FSTL1 and its downstream genes were significantly correlated within cancer cells (Figure 8D\&E). These observations suggest that FSTL1 expression is governed by both tumor intrinsic variation as well 
as TME. On the other hand, Glutamic-Pyruvic Transaminase (GPT) showed exact opposite patterns from FSTL1. The GPT expression was suppressed by hypermethylation in tumors compared to normal tissues and was associated with good survival, suggesting its role as a tumor suppressor gene (Figure 7, Supplementary Table 6). GPT is known to play an important role in the intermediary metabolism of glucose and amino acids but was reported to be associated liver diseases $^{63}$. While there were no literatures about GPT in gastric cancer, its expression was associated with good survival in liver and rectal cancer (https://www.proteinatlas.org/ENSG00000167701-GPT).

FSTL1 and GPT expression levels were regulated by its promoter methylation in gastric cancer cells (Figure 8C). Even though the expression levels of the methylation-driven key regulators in bulk tumors were significantly associated with immune or stromal proportions in TME, genes correlated with methylation-driven key regulators in GC cell lines significantly overlapped with their downstream target genes inferred using ISCT from bulk tissue data (Figures $8 \mathrm{D} \& \mathrm{E}$ ). These results suggest that tumor-TME interactions contribute to expression variations of methylation-driven key regulators such as FSTL1 and GPT, which in turn give rise to the molecular and histological heterogeneity of gastric cancer. Further investigation in cocultured system might elucidate detail roles of these genes in tumor-stroma interaction.

While our main interests are on methylation-driven key regulators, CNV alterations also play important roles in tumorigenesis and progression of GC. Using ISCT, 39 common CNVdriven key regulators were identified (details in Appendix, Supplementary Figures 9 and 10). Most of these key CNV regulators were located in chromosomes 20 and 8 where the gain of DNA copy in these locations was known in several of previous GC studies ${ }^{19,64-66}$. These genes located in the chromosome 20 were significantly amplified specifically for CIN tumors, in which no clear methylation features were associated. Indeed, no CNV-driven key regulator overlapped with the methylation-driven key regulators. Interestingly, the downstream genes of the CNV-driven key regulators were shared with those of the methylation-driven key regulators (Appendix, 
Supplementary Figure 11). These suggest the different tumorigenic pathways through methylation or copy number alterations may have similar downstream effects.

In this study, we reported 11 genes as methylation-driven key regulators identified based on ISCT. These genes characterize diverse heterogeneities of GC showing distinct molecular, cellular, and histological features as well as clinical outcomes. They were also associated with cell type proportions suggesting their roles in TME interactions. Further investigations for their molecular functions especially FSTL1 may reveal their novel roles in tumorigenesis and progression of GC that will enhance better diagnosis, prognosis, or treatment of GC patients. 


\section{Materials and Methods}

\section{GC Datasets used in integrative causal modeling}

Three GC cohorts from Hong Kong University (HKU), TCGA Stomach adenocarcinoma (TCGA), and University of Singapore (Singapore), which contain gene expression, methylation, and CNV profiles, were used in this study. The HKU dataset was deposited in European Genomephenome Archive with the study ID EGAS00001000597 ${ }^{8}$ The molecular data for the TCGA cohort $^{10}$ were downloaded from TCGA data portal (https://gdc.cancer.gov). The Singapore dataset was downloaded from Gene Expression Omnibus (GEO) with accession numbers GSE30601 ${ }^{9}$, GSE15460 ${ }^{67}$, and GSE31168 ${ }^{18}$ for methylation, gene expression and CNVs, respectively. Prior to our integrative analysis, we excluded Epstein-Barr virus (EBV) positive samples ( 6 out of 98, 24 out of 235, and 5 out of 91 in HKU, TCGA, and Singapore cohorts, respectively) according to their annotation as EBV positive GC have unique and distinct DNA hyper-methylation patterns ${ }^{68}$. Sample alignment procedure ${ }^{69}$ was applied to confirm that different types of molecular data pertaining to the same individuals were matched (details in Appendix) and 92, 211, and 86 samples in the HKU, TCGA, and Singapore datasets, respectively, were finally selected for the integrative analyses. Clinical information of the three datasets is shown in Supplementary Table 1 .

\section{Independent GC datasets for validations}

Five independent cohorts with gene expression profiles were used for validating our observations based on integrative analysis: 1) Microarray profiles of $300 \mathrm{GC}$ tumors from the Asian Cancer Research Group (ACRG) were downloaded from GEO with accession number GSE62254 ${ }^{43}$; 2) A microarray dataset of $70 \mathrm{GC}$ patients from Australian cohort (Australia) was downloaded from GEO with accession number GSE35809 ${ }^{45}$; 3) A RNAseq profiling dataset described in a proteogenomic paper by Mun et al. (CPTAC) consisting of 80 patients with early onset gastric 
cancers was downloaded from GEO with accession number GSE122401 ${ }^{70}$; 4) A microarray dataset from Yonsei hospital (Yonsei) consisting of 433 GC patient samples collected during 2000-2010 was downloaded from GEO with accession number GSE84437; 5) A microarray dataset consisting of 432 formalin-fixed paraffin-embedded (FFPE) tissues from Samsung Medical Center (SMC) was downloaded from GEO with accession number GSE26253 ${ }^{70}$. For each dataset, EBV positive tumors were removed prior to following analysis. For ACRG, Australia, and CPTAC dataset, which the EBV status of the samples was available, final 221, 69, and 74 samples were selected. For Yonsei and SMC dataset without EBV status information, samples were clustered based on gene expression of the EBV signature genes ${ }^{71}$ and no samples were filtered out in Yonsei but 57 samples were removed in SMC dataset (details in the Appendix). The demographics of these validation datasets are also listed in Supplementary Table1.

Four more gene expression datasets from the study by Oh et al. ${ }^{46}$ were additionally used to investigate the association between methylation-driven key regulators and epithelial/mesenchymal phenotypes. The processed microarray data are available in GEO with accession number GSE26899 for KUGH, GSE26901 for KUCM, GSE13861 for YUSH, GSE28541 for MDACC. The EP/MP subtype for each tumor is downloaded from supplementary tables of their paper published at Nature Communication in $2018^{46}$.

\section{Data preprocessing}

For the gene expression data in HKU, profiled on Illumina HT12v4, probe level data was obtained from median summarization over background corrected bead level data from Illumina Genome Studio, followed by quantile normalization on log (base 2) transformed probe intensity. Multiple probes for a gene were summarized (median) into gene level data after non-performing probes were excluded. For the RNAseq data from TCGA, RNA-Seq by ExpectationMaximization (RSEM) data downloaded and gene level expression was obtained from log 
transformation. For the Singapore dataset, profiled by Affymetrix UG U133A platform, probe intensity was normalized by a standard affy function, Robust Multi-array Average (RMA), with $\log$ transformation. For the five validation cohorts, we used the "getGEO" function from GEOquery package to download gene expression data as deposited in GEO database $\mathrm{e}^{72}$.

To associate DNA methylation and gene expression, we focused on methylation variations within gene promoter regions. NCBI RefSeq annotation was downloaded in gtf format and methyl probes located within 10kb upstream from Transcription Starting Sites were selected. Since the methylation values were not normally distributed as gene expression or copy numbers ${ }^{73}$, beta values of each $\mathrm{CpG}$ probe were transformed based on rank-based normal transformation using the "rntransform" function embedded in GenABEL package (Supplementary Figure 12) ${ }^{74}$.

For the CNV profiles, Circular binary segmentation $(\mathrm{CBS})^{75}$ of the $\log \mathrm{R}$ ratio values was used for all three dataset. Then each segment value was mapped to gene levels based on coordinate information of RefSeq reference annotation as mapping of methyl probes.

Detail information of molecular data platforms and the final number of features used in this study is summarized in Supplementary Table 9.

An Integrative Sequential Causality Test (ISCT) for causal regulations by DNA methylation, and CNVs

Previously, we developed a causality test for modeling transcription regulations by promoter region methylations ${ }^{34}$. As transcriptional regulations occur at multiple levels simultaneously, here we describe a model for transcriptional regulations by methylation and CNVs in both cis and trans (Figure 1A). Given a cis-regulated gene $x,\left(g_{x} \sim m_{x}+c_{x}\right)$ and a trans-regulated gene $y$, $\left(g_{y} \sim m_{x} \mid m_{y}, c_{y}\right)$, where $g_{x}$ and $g_{y}$ are expression levels, $c_{x}$ and $c_{y}$ are corresponding copy number variations, and $m_{x}$ and $m_{y}$ are promoter region methylation levels of gene $x$ and $y$, respectively, the causal relationship between cis gene expression and trans gene expression holds 
when the trans-regulation can be completely explained by the cis gene expression. In other words, we hypothesize that the trans relationship $\left(g_{y} \sim m_{x} \mid m_{y}, c_{y}\right)$ arises from the probability chain $p\left(m_{x} \rightarrow g_{x} \rightarrow g_{y} \mid c_{x}, m_{y}, c_{y}\right)$, which can be decomposed as a production of probabilities of a chain of statistical tests as below. The causal relationship is significant when the trans relationship $\left(g_{y} \sim m_{x} \mid m_{y}, c_{y}\right)$ becomes non-significant after conditioning on the cis-gene expression $g_{x}$, in which case the trans relationship between $m_{x}$ and $g_{y}$ is "caused" by $g_{x}$.

Similar to the mediation test outlined by Baron and Kenny ${ }^{36}$, the causality test can be broken down into steps: (1) the cis regulation: can be modeled as a linear regression $g_{x} \sim m_{x}+$ $c_{x}$; (2) the trans association between $g_{y}$ and $m_{x}$ : instead of being modeled as a linear regression $g_{y} \sim m_{x}+m_{y}+c_{y}$, was modeled in a sequential process: we accounted cis regulations $g_{y} \sim m_{y}+c_{y}$ and identified residual variance that could not be explained by cis regulation $g_{y}^{*}=\operatorname{residual}\left(g_{y} \sim m_{y}+c_{y}\right)$, then we modeled the trans association as $g_{y}^{*} \sim m_{x}$; (3) the association between $g_{x}$ and $g_{y}$ : was modeled similarly as $g_{y}^{*} \sim g_{x} ;$ (4) the conditional independence (the indirect effect) between $m_{x}$ and $g_{y} \mid m_{y}, c_{y}$ was modeled in a sequential procedure: identifying the residual variance that could not be explained by $g_{x}$ as $g_{y}^{* *}=$ residual $\left(g_{y}^{*} \sim g_{x}\right)$ then the conditional independence was assessed as $g_{y}^{* *} \sim m_{x}+c_{x}$ instead of a standard linear regression $g_{y} \sim m_{x}+c_{x}+g_{x}+m_{y}+c_{y}$. As we previously described causal relationship between promoter region methylation and trans gene expression ${ }^{34}, p\left(m_{x} \rightarrow g_{x} \rightarrow\right.$ $\left.g_{y} \mid c_{x}, m_{y}, c_{y}\right)$ was mainly determined by $p\left(m_{x} \perp g_{y} \mid g_{x}, m_{y}, c_{y}\right)$ given significant cis and trans relationships for gene $x$ and $y(\mathrm{FDR}<0.05)$.

Similarly, the causal relationship between a cis CNV gene $x$ and a trans gene $y$ was modeled as $p\left(c_{x} \rightarrow g_{x} \rightarrow g_{y} \mid m_{x}, m_{y}, c_{y}\right)$.

\section{Comparison with mediation tests}


A mediation test is an alternative to the ISCT for testing causal relationships between the cis gene methylation and trans gene expression. In a mediation model, the cis methylation $m_{x}$ can be perceived as the independent variable, the trans gene expression $g_{y}$ is the dependent variable, and the cis gene expression $g_{x}$ is the potential mediator. Various mediation test methods exist for testing whether the relationship between the independent variable and the dependent variable is mediated through the potential mediator. One of the most widely used approaches to test for mediation is the causal steps method ${ }^{35,36,76}$, which evaluates three regression models: the first one assesses whether the independent variable affects the mediator by regressing the mediator on the independent variable; the second one assesses whether the independent variable affects the dependent variable by regressing the dependent variable on the independent variable; the third one assesses whether the mediator affects the dependent variable when the independent variable is controlled by regressing the dependent variable on both the independent variable and the mediator. The mediation effect is established if all three regressions show significant relationships, and the effect of the independent variable on the dependent variable is reduced in its absolute size after controlling for the mediator. Moreover, if the independent variable shows no effect on the dependent variable in the third regression, the mediation is full. Another method to test for mediation is the Sobel test, ${ }^{37}$ which evaluates the significance of the mediation (indirect) effect by comparing its magnitude divided by its estiamted standard error of measurement to a normal distribution. The Sobel test is known to be conservative ${ }^{77}$ because of its normal approximation of the test statistic. Nevertheless, both mediation tests are expected to be underpowered in testing whether the association between the independent variable $m_{x}$ and the dependent variable $g_{y}$ is (completely) mediated through the mediator $g_{x}$, given that cisregulation indicates colinearity between the mediateor $g_{x}$ and the independent variable $m_{x}$. The ISCT approach, on the other hand, assigns variances to each variable according to the sequence of biological events so that it does not suffer from the colinearity problem. For example, in an 
extreme case where $g_{x}$ and $m_{x}$ are perfectly correlated, both coefficients will be non-significant in the regression $g_{y}^{*} \sim g_{x}+m_{x}$, giving non-significant result for the mediation test; whereas given significant trans-regulation between $g_{y}^{*}$ and $m_{x}$, the first regression in the ISCT approach $g_{y}^{*} \sim g_{x}$ will give significant coefficient for $g_{x}$, and the second regression $g_{y}^{* *} \sim m_{x}$ will give a non-significant coefficient for $m_{x}$, resulting in a significant case for the sequential regression approach. In reality, most of the $g_{x}$ and $m_{x}$ tested for mediation or causality are not perfectly correlated but may be highly correlated as a significant cis-regulation for the pair is required. Therefore, the mediation test is expected to give overly conservative results because of its reduced power in the presence of colinearity, while the ISCT approach is expected to be better powered in detecting the causal relationships.

\section{Simulations for comparing ISCT and mediation tests}

To estimate the power and the false postive rate of each method to detect the underlying causal/mediation relationships, we conducted two simulation studies based on a causal model and an independent model.

\section{1) Simulation \#1:}

We randomly selected 10,000 causal pairs from the HKU cohort with significant cis- and transrelationships that were tested significant by ISCT. We preserved the values of the independent variable $m_{x}$ and the potential mediator $g_{x}$ (and the covariates $c_{x}, m_{y}$ ), and simulated $g_{y}^{*}$ based on the mediator $g_{x}$ so that $g_{y}^{*}$ is correlated with $g_{x}$ at the same correlation level between $g_{x}$ and the original $g_{y}^{*}$ values in the HKU cohort, then $g_{y}$ is calculated from the simulated $g_{y}^{*}$ and its original regression coefficient. This data-generating process mimiced the effect of a mediator ${ }^{38}$, by leaving the correlation between the dependent variable and the independent variable $\operatorname{cor}\left(g_{y}\right.$, $m_{x}$ ) to vary solely as a result of the causal path from $m_{x} \rightarrow g_{x} \rightarrow g_{y}$. We selected simulated $g_{y}$ 
values showing significant trans-relationship with $m_{x}$, then applied both ISCT and two mediation tests to detect the underlying causal/mediation relationships.

\section{2) Simulation \#2:}

We randomly selected 10,000 non-causal pairs from the HKU cohort with significant cis- and trans-relationships that were tested non-significant by ISCT. We preserved the values of the independent variable $m_{x}$ and the potential mediator $g_{x}$ (and the covariate $c_{x}$ ), and simulated $m_{y}$ based on $m_{x}$ at a correlation level sampled from the correlation distribution between methylation levels of a meth-probe and that of its trans-associated gene's most associated meth-probe in the HKU cohort, then simulated the dependent variable $g_{y}$ based on $m_{y}$ at a correlation level sampled from the correlation distribution between all genes and their most associated methprobes in the HKU cohort. This data-generating process mimiced a trans relationship resulting from a path independent of $g_{x}: m_{x} \rightarrow m_{y} \rightarrow g_{y}$. We selected simulated $g_{y}$ values showing significant trans-relationship with $m_{x}$, then applied both ISCT and two mediation tests to detect the underlying causal/mediation relationships.

Furthermore, we performed two additional simulation studies given certain correlation levels among the variables to investigate the performance of each method under each specific scenario in the presence of colinearity.

\section{3) Simulation \#3:}

We randomly selected 10,000 cis-trans pairs from the HKU cohort with significant cis- and trans-relationships. We preserved the values of the independent variable $m_{x}$ (and the covariate $c_{x}$ ) and the potential mediator $g_{x}$, and generated the dependent variable $g_{y}^{*}$ so that $g_{y}^{*}$ is correlated with the mediator $g_{x}$ at given correlation levels. Then we selected cis-trans pairs and applied ISCT and two mediation tests to detect the underlying causal/mediation relationships.

\section{4) Simulation \#4:}


We randomly selected 1,000 cis-trans pairs from the HKU cohort, and preserved the values of the independent variable $m_{x}$ (and the covariate $c_{x}$ ), and simulated the potential mediator $g_{x}$ so that $g_{x}$ correlated with the independent variable $m_{x}$ at a pre-specified correlation level $\operatorname{cor}\left(g_{x}, m_{x}\right)$, then simulated the dependent variable so that the correlation between the dependent variable and the mediator at a pre-specified level. Then we selected cis-trans pairs and applied ISCT and two mediation tests to detect the underlying causal/mediation relationships.

\section{Key regulator identification}

Key regulators were determined based on the number of downstream genes. For methylationdriven key regulators, causal relationships between cis and trans genes were assessed based on individual methylation probes, summarized at gene levels as there were multiple methylation probes profiled in the promoter regions of each individual gene. Cis methylation and CNV genes were sorted based on the number of their downstream genes. Then key regulators were defined as ones whose numbers of downstream genes were significantly higher compared to others. The cutoff for defining key regulators in each dataset was set based on the reflection point from numbers of downstream genes for each regulator ${ }^{78,79}$.

\section{GC related signatures}

GC progress signatures were defined as up and down-regulated genes in advanced GCs compared to early stage ones ${ }^{50}$. GC survival associated genes were derived based on Asian Cancer Research Group (ACRG) GC cohort $^{43}$, which includes only gene expression ${ }^{67}$. Only samples with living without recurrence or samples with death due to disease were used to define survival signatures. The association of expression of each gene with survival information was tested using a Cox regression model as survival $\sim$ age + gender + expression. In total, 3375 GC survival associated genes were identified as FDR $<0.01$. 


\section{Functional analysis}

To identify enriched function among a set of selected genes, a collection of Hallmark gene sets, curated gene sets, and GO terms in Molecular Signatures Database (MSigDB) were used ${ }^{42}$. The significance of the overlap with query genes was tested via the Fisher's exact test (FET).

\section{Survival analysis}

Clinical information for samples in all datasets was downloaded from their corresponding papers or GEO database. Cancer specific survival (CSS) was available for HKU, TCGA, and ACRG datasets and recurrence free survival was used for SMC dataset. For Singapore, Australia, and Yonsei datasets, overall survival (OS) was used. CPTAC dataset was omited for survival analysis because the events occurred in only 9 samples out of 74 samples, so that it was not sufficient to perform survival analysis in the CPTAC dataset. For univariate survival analysis with age and gender as covariates was used as survival $\sim$ age + gender + factor, where factors were gene expression, cell type proportions, or clusters. R package "survival" was used for the survival analysis.

\section{Cell component decomposition}

CIBERSORT $^{80}$ (https://cibersort.stanford.edu/) was used to decompose cell components into immune, stromal and cancer proportions. For the immune references, the original LM22 data was used and proportions of individual 22 immune cell types were summed up to immune proportions. For stroma and cancer cells references, we downloaded microarray CEL files (Affymetrix HG_U133+2) of 6 stomach fibroblasts (3 submucosal and 3 subperitoneal fibroblasts) from GSE63626 $6^{81}$ and 36 stomach carcinoma cells from Cancer Cell Line Cyclopedia $(\mathrm{CCLE})^{51}$ (https://portals.broadinstitute.org/ccle). The cell profiles were processed to generate a signature matrix by comparing one cell type versus all other cell types. And the signature matrix was used to the proportions of immune, fibroblast, and cancer cells in samples of the $8 \mathrm{GC}$ 
bioRxiv preprint doi: https://doi.org/10.1101/2020.01.27.920744; this version posted January 28, 2020. The copyright holder for this preprint (which was not certified by peer review) is the author/funder, who has granted bioRxiv a license to display the preprint in perpetuity. It is made available under aCC-BY-NC-ND 4.0 International license.

cohorts (3 primary and 5 validation datasets). Cell type proportions based on DNA methylation were downloaded $\quad$ MethylCIBERSORT ${ }^{53}$ results page (https://zenodo.org/record/3242689\#.XQ0S9vlKjOR). 
bioRxiv preprint doi: https://doi.org/10.1101/2020.01.27.920744; this version posted January 28, 2020. The copyright holder for this preprint (which was not certified by peer review) is the author/funder, who has granted bioRxiv a license to display the preprint in perpetuity. It is made available under aCC-BY-NC-ND 4.0 International license.

\section{Declaration of Interests}

Seungyeul Yoo, Quan Chen, Li Wang, and Jun Zhu are employees of Sema4, a for-profit organization that promotes genomic sequencing for patient-centered healthcare. 


\section{References}

1. Bray F, Ferlay J, Soerjomataram I, et al. Global cancer statistics 2018: GLOBOCAN estimates of incidence and mortality worldwide for 36 cancers in 185 countries. CA Cancer J Clin 2018;68:394-424.

2. Rahman R, Asombang AW, Ibdah JA. Characteristics of gastric cancer in Asia. World J Gastroenterol 2014;20:4483-90.

3. Siegel RL, Miller KD, Jemal A. Cancer Statistics, 2017. CA Cancer J Clin 2017;67:7-30.

4. Pasechnikov V, Chukov S, Fedorov E, et al. Gastric cancer: prevention, screening and early diagnosis. World J Gastroenterol 2014;20:13842-62.

5. Lauren P. The Two Histological Main Types of Gastric Carcinoma: Diffuse and So-Called Intestinal-Type Carcinoma. An Attempt at a Histo-Clinical Classification. Acta Pathol Microbiol Scand 1965;64:31-49.

6. $\quad \mathrm{Hu}$ B, El Hajj N, Sittler S, et al. Gastric cancer: Classification, histology and application of molecular pathology. J Gastrointest Oncol 2012;3:251-61.

7. Correa P, Piazuelo MB. Helicobacter pylori Infection and Gastric Adenocarcinoma. US Gastroenterol Hepatol Rev 2011;7:59-64.

8. Wang K, Yuen ST, Xu J, et al. Whole-genome sequencing and comprehensive molecular profiling identify new driver mutations in gastric cancer. Nat Genet 2014;46:573-82.

9. Zouridis H, Deng N, Ivanova T, et al. Methylation subtypes and large-scale epigenetic alterations in gastric cancer. Sci Transl Med 2012;4:156ra140.

10. Cancer Genome Atlas Research N. Comprehensive molecular characterization of gastric adenocarcinoma. Nature 2014;513:202-9.

11. Mun DG, Bhin J, Kim S, et al. Proteogenomic Characterization of Human EarlyOnset Gastric Cancer. Cancer Cell 2019;35:111-124 e10.

12. Chang MS, Uozaki H, Chong JM, et al. CpG island methylation status in gastric carcinoma with and without infection of Epstein-Barr virus. Clin Cancer Res 2006;12:2995-3002.

13. Padmanabhan N, Ushijima T, Tan P. How to stomach an epigenetic insult: the gastric cancer epigenome. Nat Rev Gastroenterol Hepatol 2017;14:467-478.

14. Yoda Y, Takeshima H, Niwa T, et al. Integrated analysis of cancer-related pathways affected by genetic and epigenetic alterations in gastric cancer. Gastric Cancer 2015;18:65-76.

15. Leary RJ, Lin JC, Cummins J, et al. Integrated analysis of homozygous deletions, focal amplifications, and sequence alterations in breast and colorectal cancers. Proc Natl Acad Sci U S A 2008;105:16224-9.

16. Lee YS, Cho YS, Lee GK, et al. Genomic profile analysis of diffuse-type gastric cancers. Genome Biol 2014;15:R55.

17. Zhang D, Wang Z, Luo Y, et al. Analysis of DNA copy number aberrations by multiple ligation-dependent probe amplification on 50 intestinal type gastric cancers. J Surg Oncol 2011;103:124-32.

18. Deng N, Goh LK, Wang $\mathrm{H}$, et al. A comprehensive survey of genomic alterations in gastric cancer reveals systematic patterns of molecular 
exclusivity and co-occurrence among distinct therapeutic targets. Gut 2012;61:673-84.

19. Liang L, Fang JY, Xu J. Gastric cancer and gene copy number variation: emerging cancer drivers for targeted therapy. Oncogene 2016;35:1475-82.

20. Krepischi AC, Pearson PL, Rosenberg C. Germline copy number variations and cancer predisposition. Future Oncol 2012;8:441-50.

21. Uhlik MT, Liu J, Falcon BL, et al. Stromal-Based Signatures for the Classification of Gastric Cancer. Cancer Res 2016;76:2573-86.

22. Puleo F, Nicolle R, Blum Y, et al. Stratification of Pancreatic Ductal Adenocarcinomas Based on Tumor and Microenvironment Features. Gastroenterology 2018;155:1999-2013 e3.

23. Erdag G, Schaefer JT, Smolkin ME, et al. Immunotype and immunohistologic characteristics of tumor-infiltrating immune cells are associated with clinical outcome in metastatic melanoma. Cancer Res 2012;72:1070-80.

24. Thorsson V, Gibbs DL, Brown SD, et al. The Immune Landscape of Cancer. Immunity 2018;48:812-830 e14.

25. Fluxa P, Rojas-Sepulveda D, Gleisner MA, et al. High CD8(+) and absence of Foxp3(+) T lymphocytes infiltration in gallbladder tumors correlate with prolonged patients survival. BMC Cancer 2018;18:243.

26. Pardoll DM. The blockade of immune checkpoints in cancer immunotherapy. Nat Rev Cancer 2012;12:252-64.

27. Jamal-Hanjani M, Quezada SA, Larkin J, et al. Translational implications of tumor heterogeneity. Clin Cancer Res 2015;21:1258-66.

28. Binnewies M, Roberts EW, Kersten $\mathrm{K}$, et al. Understanding the tumor immune microenvironment (TIME) for effective therapy. Nat Med 2018;24:541-550.

29. Zunder SM, van Pelt GW, Gelderblom HJ, et al. Predictive potential of tumourstroma ratio on benefit from adjuvant bevacizumab in high-risk stage II and stage III colon cancer. Br J Cancer 2018;119:164-169.

30. Kemi N, Eskuri M, Herva A, et al. Tumour-stroma ratio and prognosis in gastric adenocarcinoma. Br J Cancer 2018;119:435-439.

31. Peng C, Liu J, Yang G, et al. The tumor-stromal ratio as a strong prognosticator for advanced gastric cancer patients: proposal of a new TSNM staging system. J Gastroenterol 2018;53:606-617.

32. Wu Y, Grabsch $\mathrm{H}$, Ivanova $\mathrm{T}$, et al. Comprehensive genomic meta-analysis identifies intra-tumoural stroma as a predictor of survival in patients with gastric cancer. Gut 2013;62:1100-11.

33. Veenstra VL, Damhofer H, Waasdorp C, et al. ADAM12 is a circulating marker for stromal activation in pancreatic cancer and predicts response to chemotherapy. Oncogenesis 2018;7:87.

34. Yoo S, Takikawa S, Geraghty $P$, et al. Integrative analysis of DNA methylation and gene expression data identifies EPAS1 as a key regulator of COPD. PLoS Genet 2015;11:e1004898.

35. MacKinnon DP, Fairchild AJ, Fritz MS. Mediation analysis. Annu Rev Psychol 2007;58:593-614. 
36. Baron RM, Kenny DA. The moderator-mediator variable distinction in social psychological research: conceptual, strategic, and statistical considerations. J Pers Soc Psychol 1986;51:1173-82.

37. Sobel M. Asymptotic confidence intervals for indirect effects in structural equation models. Sociological methodology 1982;13:290-312.

38. Fiedler K, Schott M, Meiser T. What mediation analysis can (not) do. Journal of Experimental Social Psychology 2011;47:1231-1236.

39. Chandanos E, Lagergren J. Oestrogen and the enigmatic male predominance of gastric cancer. Eur J Cancer 2008;44:2397-403.

40. Ur Rahman MS, Cao J. Estrogen receptors in gastric cancer: Advances and perspectives. World J Gastroenterol 2016;22:2475-82.

41. Liberzon A, Birger C, Thorvaldsdottir H, et al. The Molecular Signatures Database (MSigDB) hallmark gene set collection. Cell Syst 2015;1:417-425.

42. Subramanian A, Tamayo P, Mootha VK, et al. Gene set enrichment analysis: a knowledge-based approach for interpreting genome-wide expression profiles. Proc Natl Acad Sci U S A 2005;102:15545-50.

43. Cristescu R, Lee J, Nebozhyn M, et al. Molecular analysis of gastric cancer identifies subtypes associated with distinct clinical outcomes. Nat Med 2015;21:449-56.

44. Cislo M, Filip AA, Arnold Offerhaus GJ, et al. Distinct molecular subtypes of gastric cancer: from Lauren to molecular pathology. Oncotarget 2018;9:19427-19442.

45. Lei Z, Tan IB, Das $\mathrm{K}$, et al. Identification of molecular subtypes of gastric cancer with different responses to PI3-kinase inhibitors and 5-fluorouracil. Gastroenterology 2013;145:554-65.

46. Oh SC, Sohn BH, Cheong JH, et al. Clinical and genomic landscape of gastric cancer with a mesenchymal phenotype. Nat Commun 2018;9:1777.

47. Chen D, Cao G, Qiao C, et al. Alpha B-crystallin promotes the invasion and metastasis of gastric cancer via NF-kappaB-induced epithelial-mesenchymal transition. J Cell Mol Med 2018;22:3215-3222.

48. Kudo-Saito C, Ishida A, Shouya Y, et al. Blocking the FSTL1-DIP2A Axis Improves Anti-tumor Immunity. Cell Rep 2018;24:1790-1801.

49. Demirag GG, Sullu Y, Gurgenyatagi D, et al. Expression of plakophilins (PKP1, PKP2, and PKP3) in gastric cancers. Diagn Pathol 2011;6:1.

50. Vecchi M, Nuciforo P, Romagnoli S, et al. Gene expression analysis of early and advanced gastric cancers. Oncogene 2007;26:4284-94.

51. Barretina J, Caponigro G, Stransky N, et al. The Cancer Cell Line Encyclopedia enables predictive modelling of anticancer drug sensitivity. Nature 2012;483:603-7.

52. Ghandi M, Huang FW, Jane-Valbuena J, et al. Next-generation characterization of the Cancer Cell Line Encyclopedia. Nature 2019;569:503-508.

53. Chakravarthy A, Furness A, Joshi K, et al. Pan-cancer deconvolution of tumour composition using DNA methylation. Nat Commun 2018;9:3220.

54. An C, Choi IS, Yao JC, et al. Prognostic significance of CpG island methylator phenotype and microsatellite instability in gastric carcinoma. Clin Cancer Res 2005;11:656-63. 
55. Shigeyasu K, Nagasaka T, Mori Y, et al. Clinical Significance of MLH1 Methylation and CpG Island Methylator Phenotype as Prognostic Markers in Patients with Gastric Cancer. PLoS One 2015;10:e0130409.

56. Mishra P, Tang W, Putluri V, et al. ADHFE1 is a breast cancer oncogene and induces metabolic reprogramming. J Clin Invest 2018;128:323-340.

57. Hao S, Yu J, He W, et al. Cysteine Dioxygenase 1 Mediates Erastin-Induced Ferroptosis in Human Gastric Cancer Cells. Neoplasia 2017;19:1022-1032.

58. Harada H, Hosoda K, Moriya H, et al. Cancer-specific promoter DNA methylation of Cysteine dioxygenase type 1 (CD01) gene as an important prognostic biomarker of gastric cancer. PLoS One 2019;14:e0214872.

59. Chan QK, Ngan HY, Ip PP, et al. Tumor suppressor effect of follistatin-like 1 in ovarian and endometrial carcinogenesis: a differential expression and functional analysis. Carcinogenesis 2009;30:114-21.

60. Ni X, Cao X, Wu Y, et al. FSTL1 suppresses tumor cell proliferation, invasion and survival in non-small cell lung cancer. Oncol Rep 2018;39:13-20.

61. Liu Y, Han X, Yu Y, et al. A genetic polymorphism affects the risk and prognosis of renal cell carcinoma: association with follistatin-like protein 1 expression. Sci Rep 2016;6:26689.

62. Mashimo J, Maniwa R, Sugino H, et al. Decrease in the expression of a novel TGF beta1-inducible and ras-recision gene, TSC-36, in human cancer cells. Cancer Lett 1997;113:213-9.

63. Pagliarini R, Castello R, Napolitano F, et al. In Silico Modeling of Liver Metabolism in a Human Disease Reveals a Key Enzyme for Histidine and Histamine Homeostasis. Cell Rep 2016;15:2292-2300.

64. Jin DH, Park SE, Lee J, et al. Copy Number Gains at 8q24 and 20q11-q13 in Gastric Cancer Are More Common in Intestinal-Type than Diffuse-Type. PLoS One 2015;10:e0137657.

65. Cheng L, Wang $\mathrm{P}$, Yang S, et al. Identification of genes with a correlation between copy number and expression in gastric cancer. BMC Med Genomics 2012;5:14.

66. Hudler P. Genetic aspects of gastric cancer instability. ScientificWorldJournal 2012;2012:761909.

67. Ooi $\mathrm{CH}$, Ivanova $\mathrm{T}, \mathrm{Wu} \mathrm{J}$, et al. Oncogenic pathway combinations predict clinical prognosis in gastric cancer. PLoS Genet 2009;5:e1000676.

68. Matsusaka K, Kaneda A, Nagae G, et al. Classification of Epstein-Barr viruspositive gastric cancers by definition of DNA methylation epigenotypes. Cancer Res 2011;71:7187-97.

69. Yoo S, Huang T, Campbell JD, et al. MODMatcher: multi-omics data matcher for integrative genomic analysis. PLoS Comput Biol 2014;10:e1003790.

70. Lee J, Sohn I, Do IG, et al. Nanostring-based multigene assay to predict recurrence for gastric cancer patients after surgery. PLoS One 2014;9:e90133.

71. Gulley ML. Genomic assays for Epstein-Barr virus-positive gastric adenocarcinoma. Exp Mol Med 2015;47:e134.

72. Davis S, Meltzer PS. GEOquery: a bridge between the Gene Expression Omnibus (GEO) and BioConductor. Bioinformatics 2007;23:1846-7. 
73. Goh L, Yap VB. Effects of normalization on quantitative traits in association test. BMC Bioinformatics 2009;10:415.

74. Aulchenko YS, Ripke S, Isaacs A, et al. GenABEL: an R library for genome-wide association analysis. Bioinformatics 2007;23:1294-6.

75. Venkatraman ES, Olshen AB. A faster circular binary segmentation algorithm for the analysis of array CGH data. Bioinformatics 2007;23:657-63.

76. Judd CM, Kenny DA. Process Analysis: Estimating Mediation in Treatment Evaluations. Evaluation Review 1981;5:602-619.

77. Mackinnon DP, Warsi G, Dwyer JH. A Simulation Study of Mediated Effect Measures. Multivariate Behav Res 1995;30:41.

78. Loven J, Hoke HA, Lin CY, et al. Selective inhibition of tumor oncogenes by disruption of super-enhancers. Cell 2013;153:320-34.

79. Whyte WA, Orlando DA, Hnisz D, et al. Master transcription factors and mediator establish super-enhancers at key cell identity genes. Cell 2013;153:307-19.

80. Newman AM, Liu CL, Green MR, et al. Robust enumeration of cell subsets from tissue expression profiles. Nat Methods 2015;12:453-7.

81. Higuchi Y, Kojima M, Ishii G, et al. Gastrointestinal Fibroblasts Have Specialized, Diverse Transcriptional Phenotypes: A Comprehensive Gene Expression Analysis of Human Fibroblasts. PLoS One 2015;10:e0129241. 


\section{Figure Legends}

\section{Figure 1. Overview of the study}

A. ISCT: Given, cis- and trans- relationship among DNA methylation, CNV, and gene expression, the causal relationship between methylation status of gene $i\left(m_{i}\right)$ and expression of gene $j\left(g_{i}\right)$ is tested. The local methylation and CNV status of trans genes are also considered in the model using $g_{j}^{*}$ instead of $g_{j}$.

B. The definition of key regulator $x$ : A cis gene $x$ causally regulates significantly larger number of downstream genes above cutoff determined from a tangent line.

C. Overall procedures of meta-analysis using ISCT and cell type deconvolution analysis from the three integrative datasets as well as the five gene-expression validation datasets.

Figure 2. Simulation results to compare ISCT to mediation test

A. The true positive rate of each test estimated by the proportion of simulated causal pairs tested significant.

B. For each pre-specified correlation level between $g_{x}$ and $g_{y}^{*}$, the dashed line shows the proportion of the simulated $g_{y}^{*}$ 's showing significant trans relationship with $m_{x}$; the yellow line shows the proportion detected by the Sobel mediation test with significant mediated relationship; the blue line shows the proportion detected by the mediation method with significant mediated relationship; the red line shows the proportion detected by the ISCT method with significant causal relationship.

C. The pairwise correlation between $m_{x}, g_{x}$ and $g_{y}^{*}$ among 1) all the cis-trans gene pairs tested, 2) the significant pairs detected by the ISCT method, 3) the significant pairs detected by the mediation test, 4) the pairs detected by the ISCT method only, and 5) the pairs detected by the mediation test only.

D. For each combination of a pre-specified $\operatorname{cor}\left(g_{x}, m_{x}\right)$ and $\operatorname{cor}\left(g_{y}^{*}, g_{x}\right)$, the proportion of simulated $g_{x}, g_{y}^{*}$ pairs with both significant cis and trans relationships with $m_{x}$, and out of which the proportion detected by each method with significant causal/mediation relationship.

\section{Figure 3. Identification of methylation-driven key regulators using ISCT}

A. Comparison of the number of cis methylation genes identified from three GC cohorts.

B. Comparison of the number of trans methylation pairs identified from three GC cohorts.

C. Comparison of the directions of trans association of common trans genes among three GC cohorts.

D. Identification of methylation-driven key regulators using cutoffs determined based on number of downstream genes in each GC cohorts.

E. Common methylation-driven key regulators identified from three GC cohorts.

Figure 4. Tumor clusters based on the gene expression levels of the 11 methylation-driven key regulators

A. Co-expression of the 11 methylation-driven key regulators in all GC cohorts was measured as Pearson correlation coefficients. CDO1, CRYAB, FSTL1, and ADHFE1 were clustered in one group (G1), RHOH and PTPRCAP in another (G2) and the rest (GPT, $S O R D, P K P 3, R A B 25$, and $S F N$ ) in the other group (G3). In the SMC datasets, $R H O H$ was not profiled and NA values were colored in white. 
B. K-mean clustering of GC tumors $(\mathrm{k}=3)$ based on expression levels of the methylationdriven key regulators.

C. The proportion of molecular subtypes, Lauren class, and tumor stages within the tumor clusters. The molecular subtypes were determined in the original study of each cohort. For the Yonsei and SMC datasets, only stage information was available.

D. KM-plots for survival analysis (Overall survival) among the tumor clusters. The number of samples in each cluster is shown. Survival differences between C1 and C3 were measured as likelihood ratio test p-values. For the HKU datasets, patients with palliative treatments were removed for survival analysis.

Figure 5. Tumor clusters based the 11 methylation-driven key regulators overlap with epithelial/mesenchymal phenotypes

A. Comparison of the tumor clusters based on the 11 methylation-driven key regulators (Figure 4B) with Epithelial/Mesenchymal subtypes determined in Oh et al.'s report ${ }^{46}$. Barplot showing overlapping rate (Supplementary Table 5) between two clustering results in TCGA and ACRG datasets.

B. K-mean clustering of $\mathrm{GC}$ tumors $(\mathrm{k}=3)$ based on expression levels of the methylationdriven key regulators for 4 datasets not included in our study (KUGH, YUSH, KUCM, and MDACC).

C. Comparison of the tumor clusters from Figure 7B with Epithelial/Mesenchymal subtypes determined in Oh et al.'s report ${ }^{46}$.

D \& E. Kaplan-Meier plots showing overall survival (D) and recurrence free survival (E) of each cluster. P-values indicate the significance of survivals between $\mathrm{C} 1$ and $\mathrm{C} 3$. Recurrence free survival for MDACC was not available.KM-plots for survival analysis (Overall survival) among the tumor clusters. The number of samples in each cluster is shown. Survival differences between C1 and C3 were measured as likelihood ratio test pvalues.

Figure 6. Adjuvant chemotherapy (CTX) sensitivity depending on tumor subtypes as well as tumor stages.

A. Survival differences between patients with and without CTX in each group. C1-C3 tumors based on our clustering method and EP and MP subtypes reported by Oh et al. ${ }^{46}$

B. Survival differences among different tumor stages (II, III, and IV) in each group.

C. Association between CTX and progression free survival at stage II.

D. Association between CTX and progression free survival at stage III.

E. Association between CTX and progression free survival at stage IV.

Figure 7. Associations between the methylation-driven key regulators and disease phenotypes

A. Univariate survival analysis based on expression of the 11 methylation-driven key regulators in 7 datasets. Hazard ratios with $95 \%$ confidence intervals were measured with corresponding p-value (Methods). The significant association is marked in red for poor prognosis and in blue for good prognosis.

B. Association of expression of the 11 methylation-driven key regulators with tumor stages in 7 datasets. The coefficient of expression of each key regulator in a regression model stage $\sim$ age + gender + expression was measured with standard errors. The significant association is marked in red for advanced and in blue for early stages.

C. The downstream genes were also compared with disease signatures such as survivalassociated genes from ACRG dataset and progression signatures from Vecchi et al. (Methods). Here, the associations with signatures with good prognostics are shown in blue while the ones with bad prognostics are in red. 
Figure 8. Tumor intrinsic variations associated with the methylation-driven key regulators

A. Distribution of gene expression $(\log 2(\mathrm{RSEM}))$ of the methylation-driven key regulators in 36 CCLE gastric cancer cells.

B. Distribution of DNA methylation level ( $\beta$-value) within $1 \mathrm{~kb}$ from TSS of the 11 methylation-driven key regulators in 33 CCLE gastric cancer cells. $\beta$-values for $R H O H$ and $R A B 25$ were not available.

C. Four methylation-driven key regulators (ADHFE1, FSTL1, GPT, and SFN) showing significant correlation between promoter methylation and gene expression in 33 CCLE gastric cancer cells. Spearman correlation coefficients (rho) with p-values are shown.

D. Association between downstream genes identified based on ISCT and co-expressed genes for 4 methylation-driven key regulators (FSTL1, ADHFE1, GPT, and SFN) in CCLE gastric cancer cells. The association strengths were measured by Odd Ratios and p-value (-log10) from FET using common genes between CCLE and our three primary datasets for ISCT as gene universe $(\mathrm{N}=14581)$.

E. For the 4 methylation-driven key regulators, the correlation coefficients of their downstream genes in CCLE cells were compared with those of the background genes. The red indicates density for positively regulated downstream and the blue for negatively regulated downstream genes. Gray indicates distribution of correlation coefficients of background genes. The number indicates the number of downstream genes for each key regulator covered in CCLE RNAseq data. 


\section{Expanded View Figure legends}

Figure EV1. Downstream target genes of the methylation-driven key regulators

A. The overlap of downstream genes of the 11 methylation-driven key regulators. The significance of the overlap was measured by FET p-value (-log10(p-value)).

B. The downstream genes were compared with MSigDB Hallmark gene sets and significantly associated gene sets $(\mathrm{p}<0.001$ from multiple testing) with any downstream gene sets are shown.

Figure EV2. Gene expression difference of the methylation-driven key regulators in tumors compared to normal tissues.

The expression levels of the methylation-driven key regulators were compared between normal and tumors based on Student t-test and Wilcox Rank Sum test A. in TCGA (211 tumors vs. 27 normal tissues) and B. in HKU (92 tumor vs. 35 normal tissues). The significant differences $(\mathrm{p}<0.01)$ are marked in red. Normal tissues are not available for Singapore dataset.

Figure EV3. Comparison of cell types proportions based on DNA methylation (MethylCIBERSORT) and gene expression (CIBERSORT).

The cell type proportions of 211 TCGA samples were measured based on DNA methylation and gene expression were compared. Pearson correlation and corresponding p-values were measured for immune, stromal, and cancer proportions. 
A

${ }^{\mathrm{aw}} \mathrm{C}$

ISCT

$p\left(m_{x}->g_{x}->g_{y} \mid\right.$ cis, trans $)$

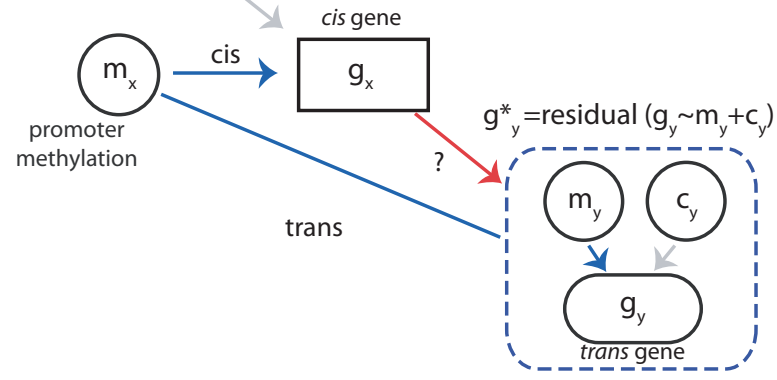

Key regulator $x$

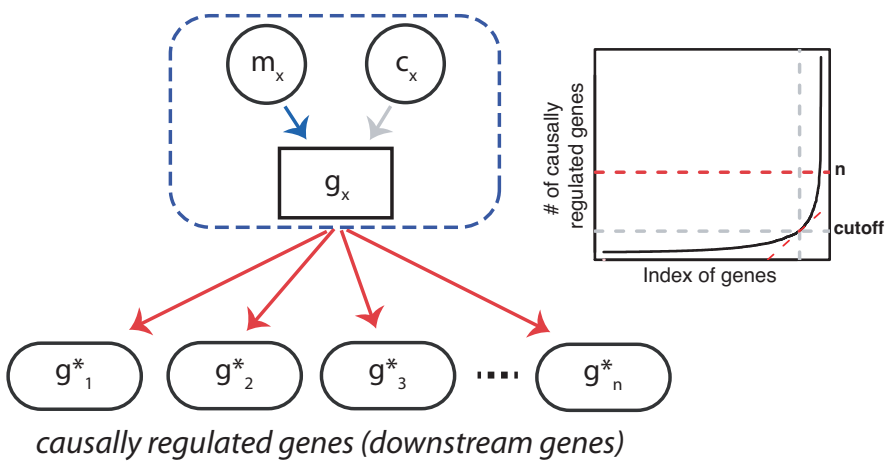

C

Integrative

dataset

ISCT

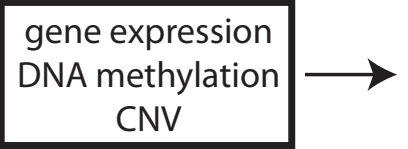

1) cis associated genes

2) trans associated genes

3) causality test

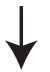

methylation-driven

key regulators

dataset

gene expression

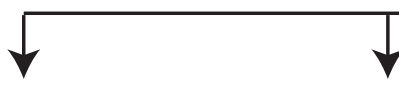

Functional analysis (downstream genes)

MsigDB

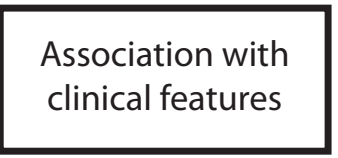

Clinical significance
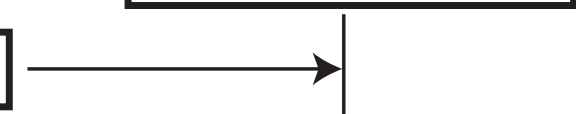
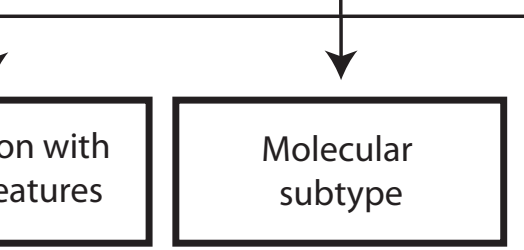

Unsuperised clustering

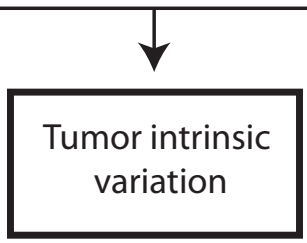

CCLE

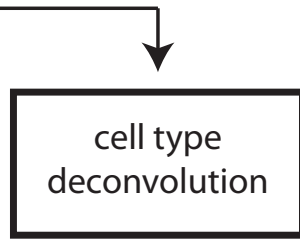

CIBERSORT 
bioRxiv preprint doi: http $\Omega$ doi.org/10.1101/2020.01.27.920744; this veston posted January 28,2020 . The copyright holder for this preprint (which was not certified byeer review) is the author/funder, who has granted bioRxiv a license to display the preprint in perpetuity. It is made Causal Model
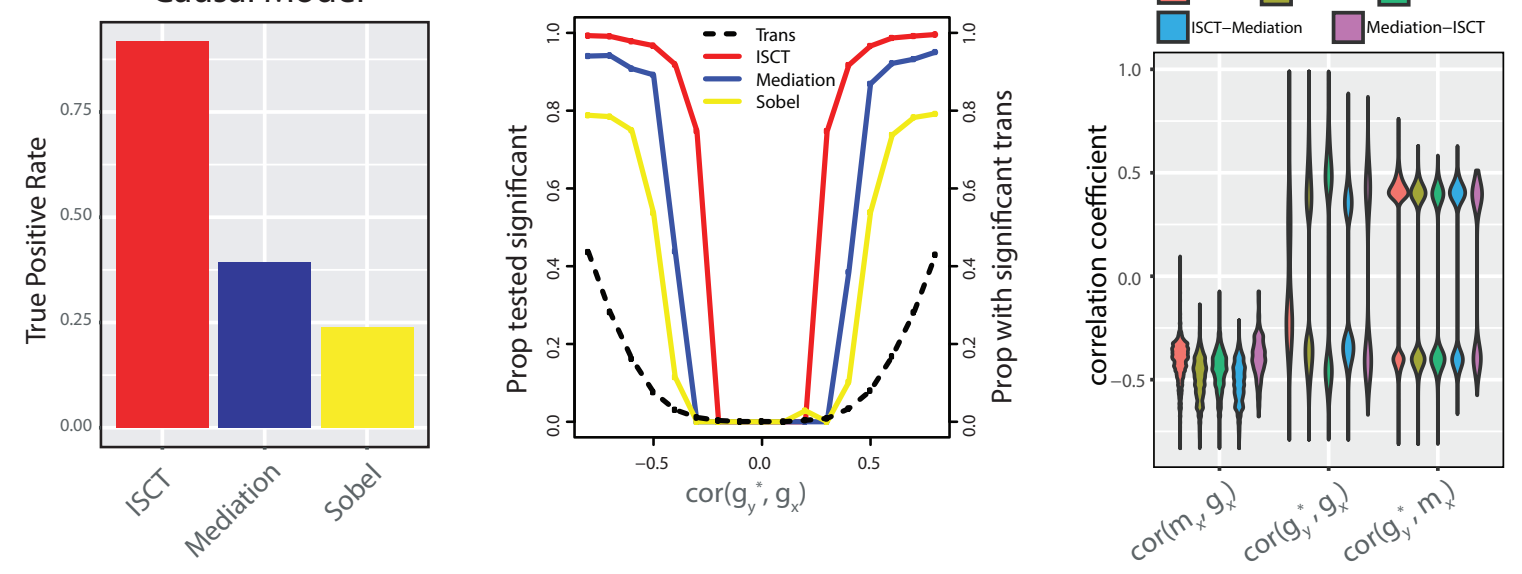

D

Trans \& cis

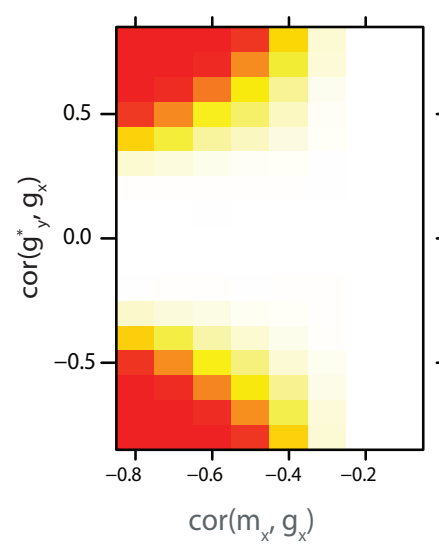

ISCT

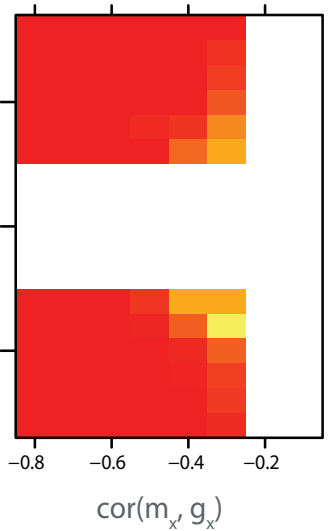

Mediation

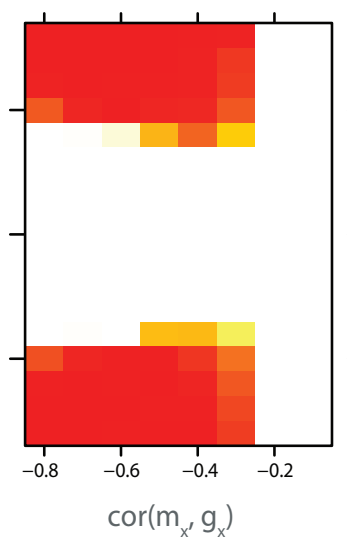

Sobel

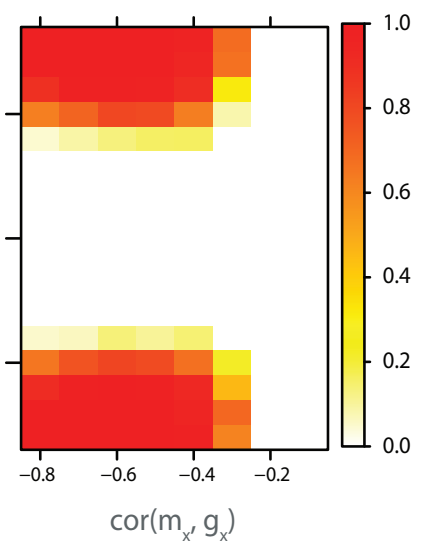


A cis methytion genes

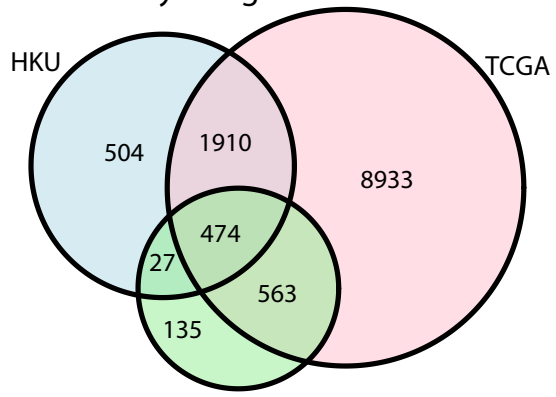

Singapore

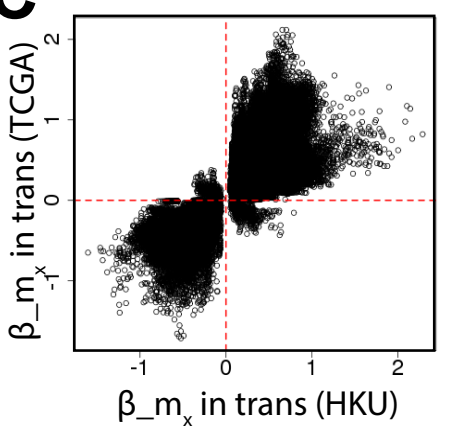

D

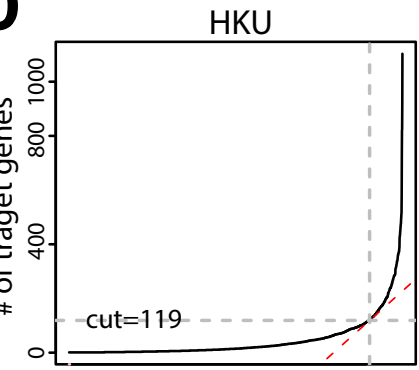

Index of regulators
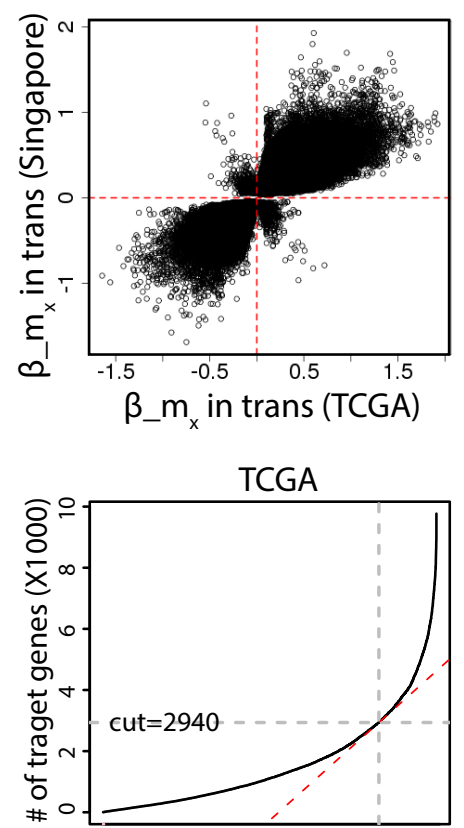

Index of regulators trans methytion pairs

HKU

387409
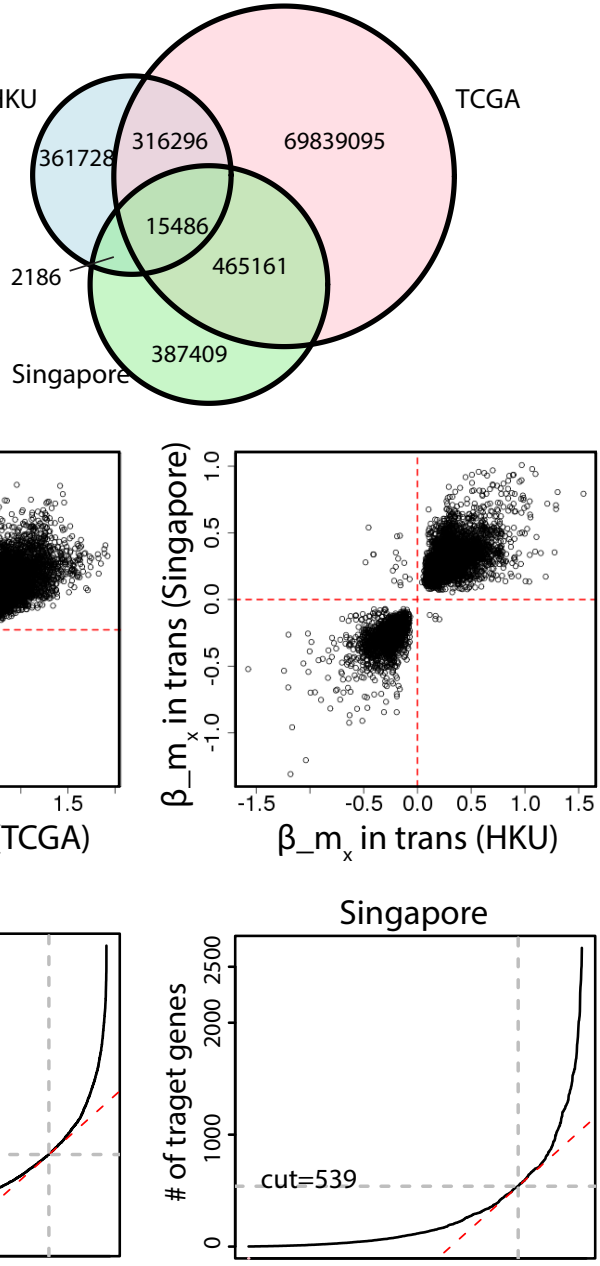

Index of regulators

ACTA2, ACTG2, AGTR1, ANGPTL2, ARHGAP27, ARHGAP30, ASB2, ATP8B2, BCAP31, BVES, C16orf45, C1QTNF3, CALML4, CBLC, CCL20, CDCP1, CMTM3, CNKSR1, CPNE8, DAAM2, DDR2, DENND2D, DES, DOK5, EFS, ELF3, ELMO1, ELOVL4, EMILIN1, EPHA7, EVL, FAAH, FGFR1, FRK, FRZB, GAS2L3, GAS7, GPRASP1, GPT2, GPX7, GSTM5, GYPC, HNF4A, HOXB2, HSPB2, HTATIP2, IGF1, ILIRN, ISLR, LAMB3, LDB3, LDOC1, LGI4, LRRC8E, LY86, MAB21L2, MAMDC2, MAP1A, MAP7, MAPK13, MEG3, MEOX1, MFAP4, MRGPRF MRVI1, MYOCD, NDN, NNAT, NPR2, OLFML3, PCDHB15, PDLIM4, PDZK1IP1, PDZRN4, PEG3, PENK, PHLDA2, PKIA, PLEKHG6, PNKD, POPDC2, PPP1R16A, PTPN13, PTPRH, PTPRM, RASL12, RTKN, S100A14, S100A16, SCRG1, SFRP1, SFRP2, SH2D3C SLC22A18AS, SLC25A5, SLC39A4, SLIT2, SMPX, SOX17, SPG20, SPINT2, ST6GALNAC5, STAP2, STMN2, TACR2, TCF21, TCF4, TEX264, THBS4, TMEM88, TNS1, TOM1L1, TPM1, TRAF1, TRAM1L1, TUSC3, UCHL1, ZNF354C, ZNF415, ZNF667, ZNF671, ZNF677

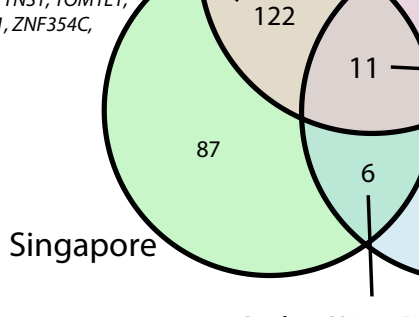

ADAP1, ADCY4, ANTXR2, BCL2L15,BRCA1, C1R, C1orf162, CDCA5, CDCA8, CENPN, CEP152, CFLAR, CHFR, CHTF18, CSAG1, DBNDD1, DDR1, DUS3L, EFNA1, ENO1, EPM2AIP1, EVI2A, EVI2B, FAM64A, FAM83H, FGF2, GAS8, GINS2, GOLT1A, GRB7, GRHL2, HMGA1, HMGB2, HOXA10, IFFO1, INF2, \begin{tabular}{l} 
TCGA INPP1, JUP, KCNQ1OT1, KIAA0907, KIF15, LAX1, LRRC61, LTC4S, \\
\hline
\end{tabular} LYL1, MAL2, MEF2D, MGAT1, MLH1, MSH5, MST1R, MXRA7, MYO1G, NCBP2, NUP43, PCSK9, PDIA6, POU2AF1, PPP1R16B, $823 \quad P P P 1 R 1 B, P R A M E, P R C 1, P S M B 8, P S M B 9, P Y C R L, R A C 3, R C O R 2$, SLC44A4, SNORA41, SNRPG, SNTB1, SOAT2, SPAG5, SPINT1, STMN1, TACC3, TBC1D10C, TMEM177, TOP1MT, TRAIP, UNC93B1, USP5, VANGL2, ZNF695, ZSCAN18 (1) 


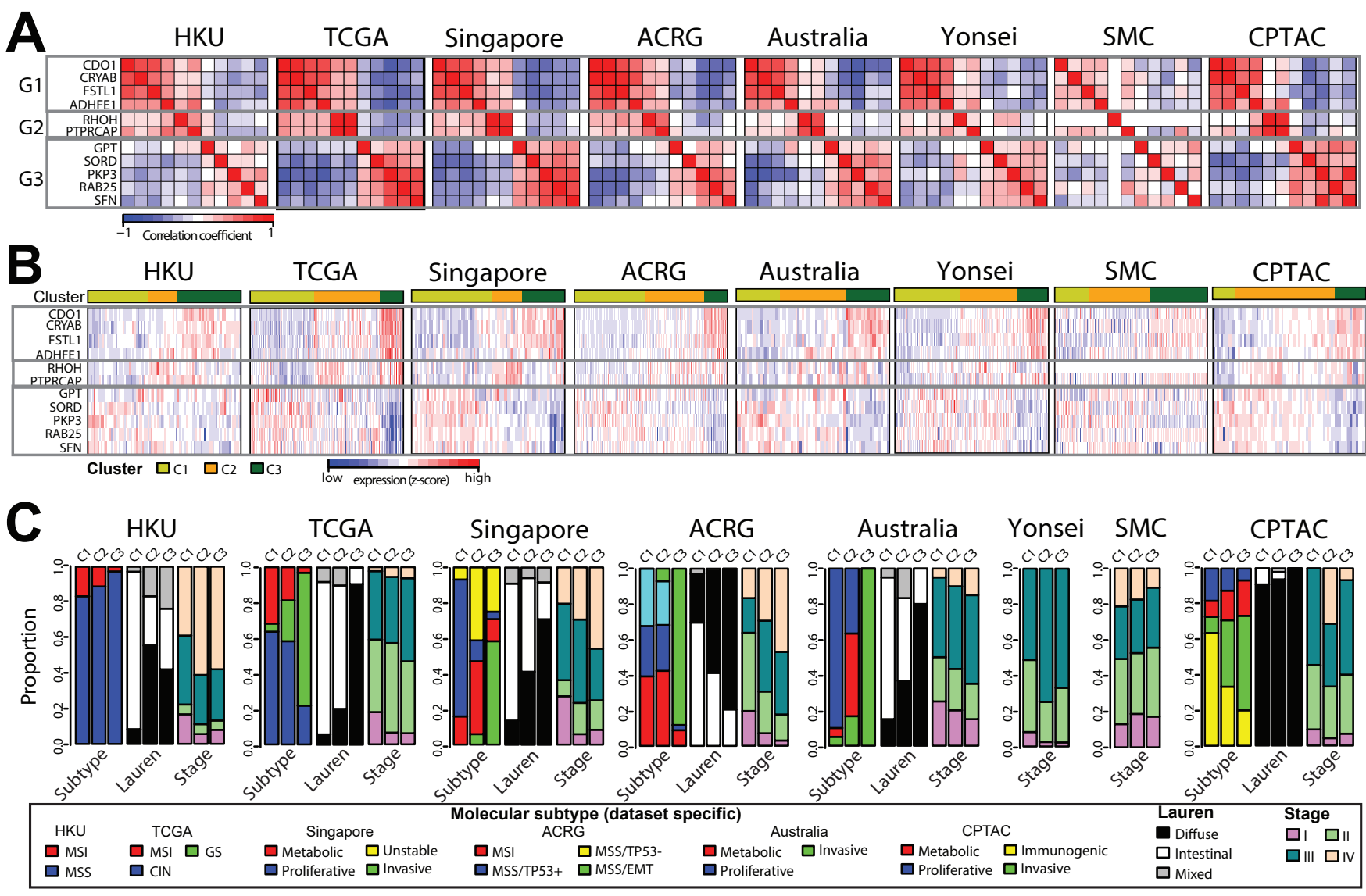

D
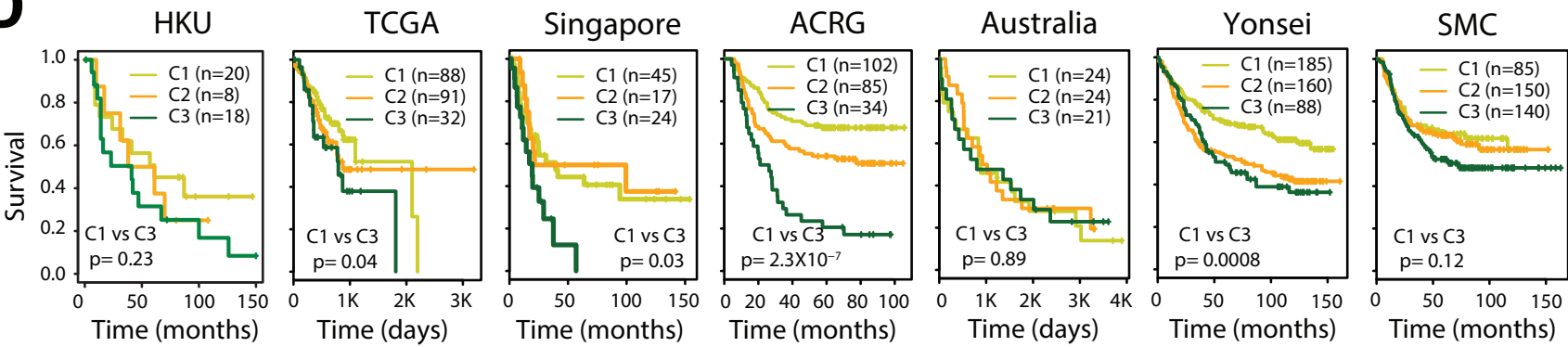


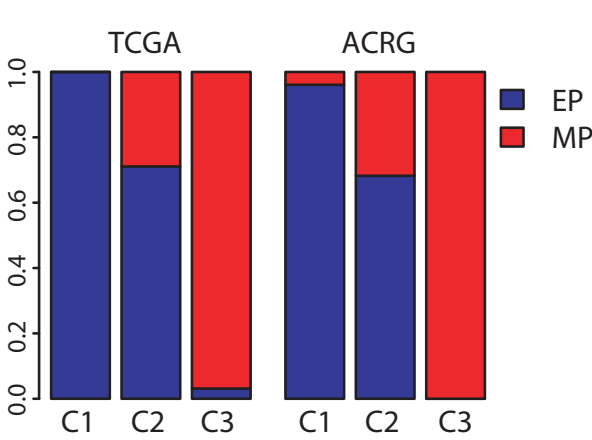

C
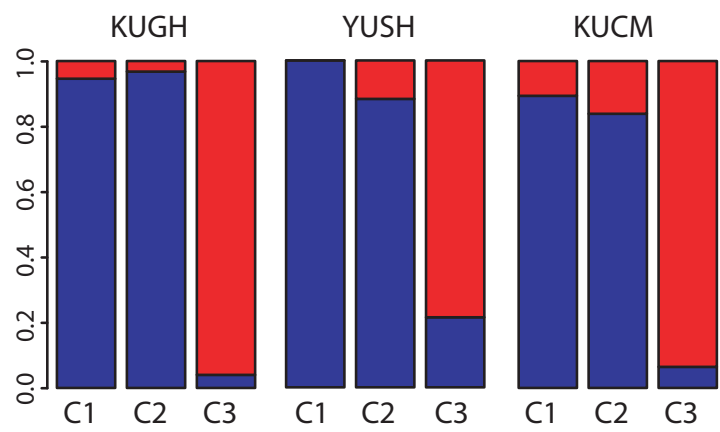

MDACC

B

KUGH

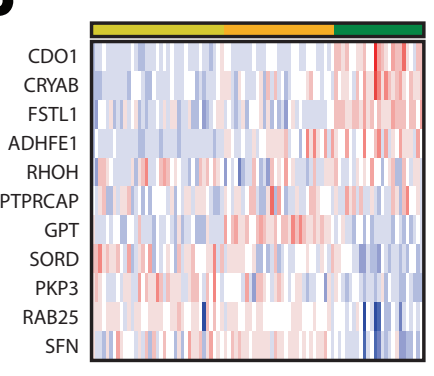

YUSH

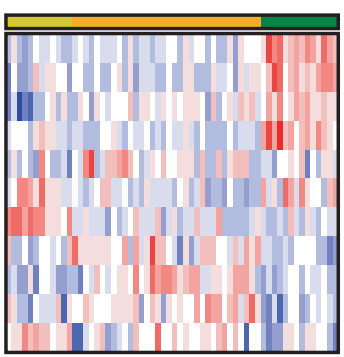

KUCM

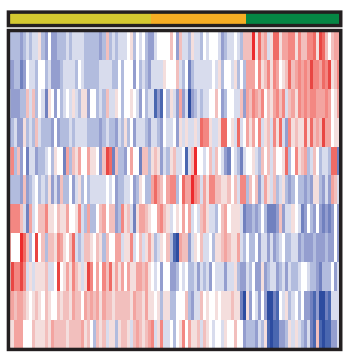

MDACC

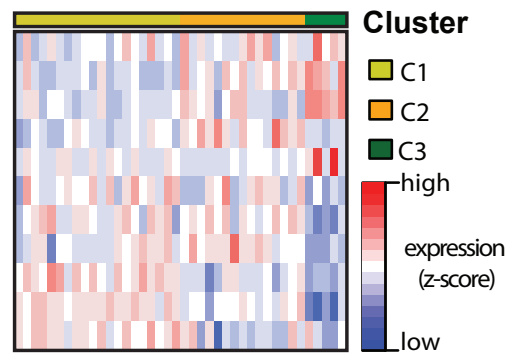

D

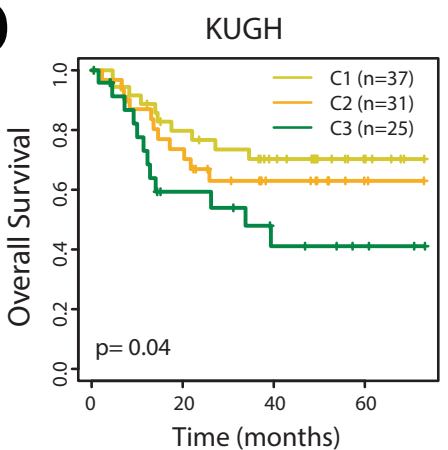

E

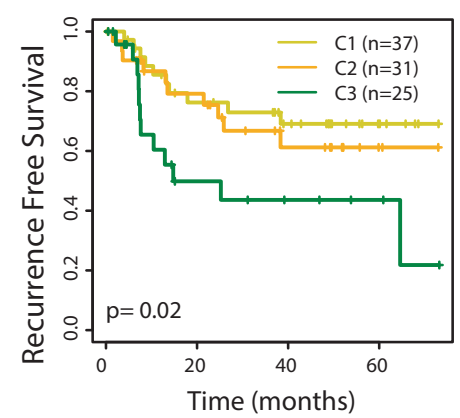

KUGH
YUSH

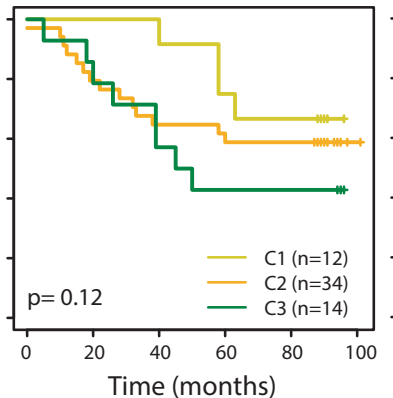

Time (months)
KUCM

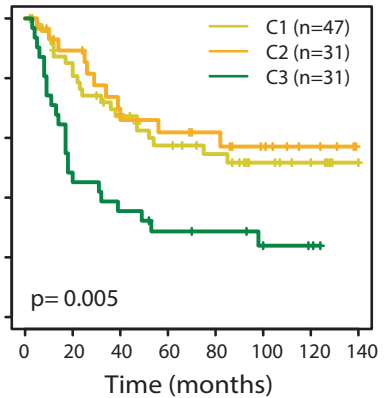

Time (months)
MDACC

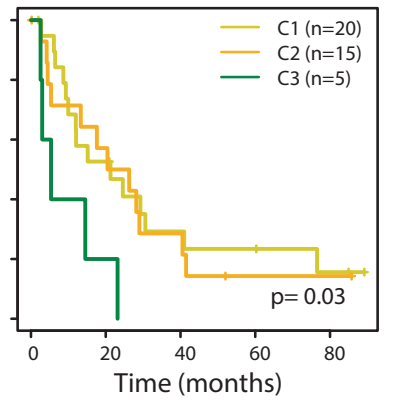

YUSH

KUCM

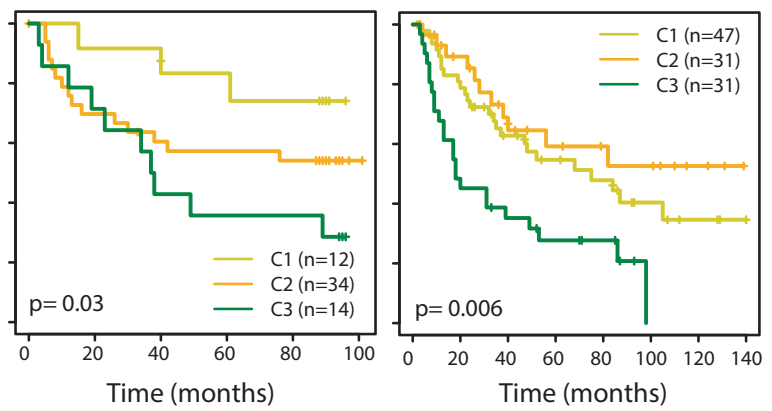



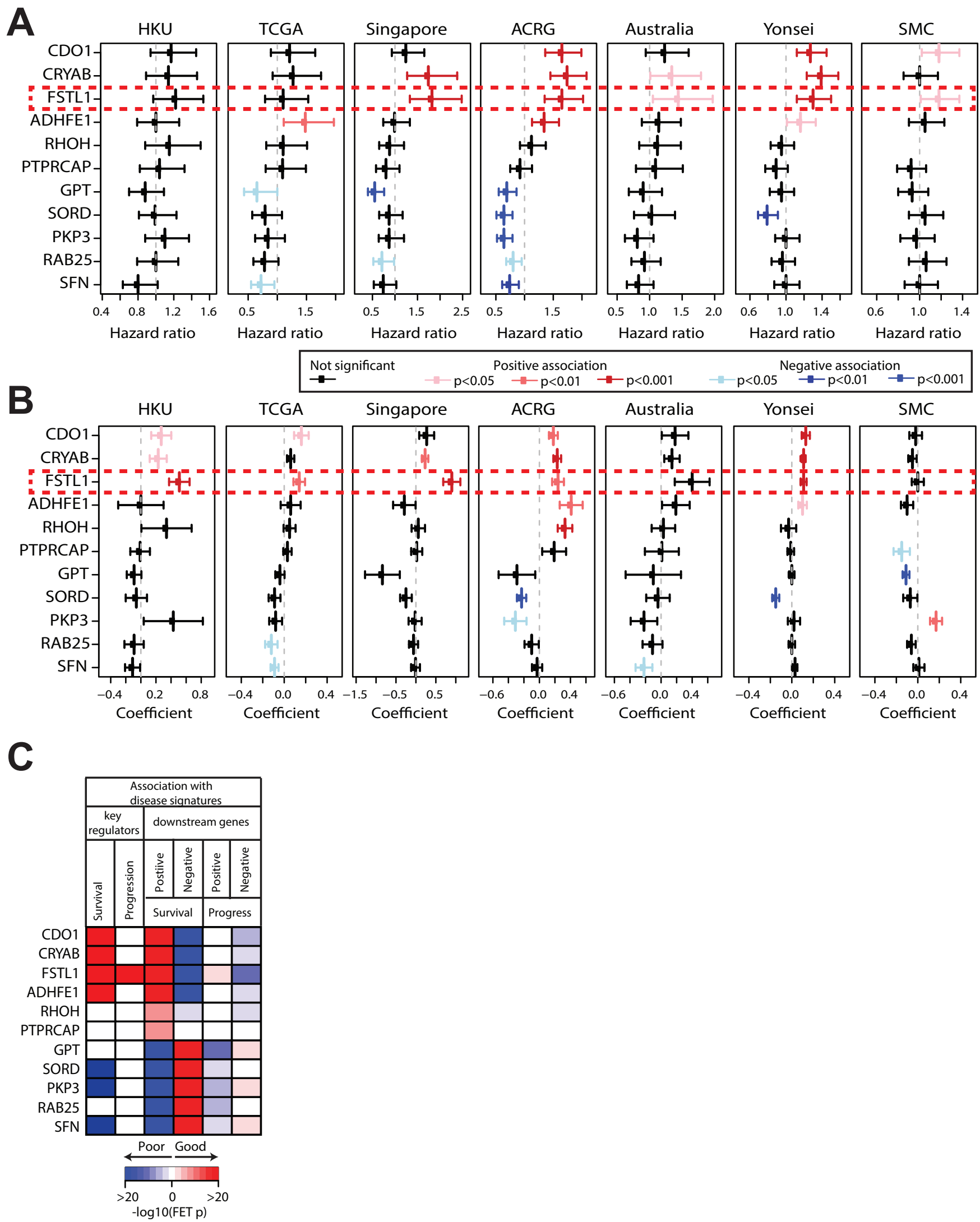
bioRxiv preprint doi: https://doi,org/10.1101/2020.01.27.920744; thiswersion posted January $28,2220$. The copyright holder for this preprint

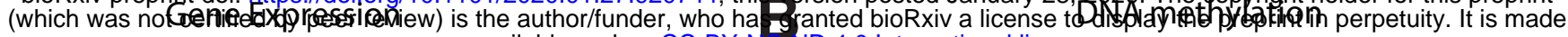
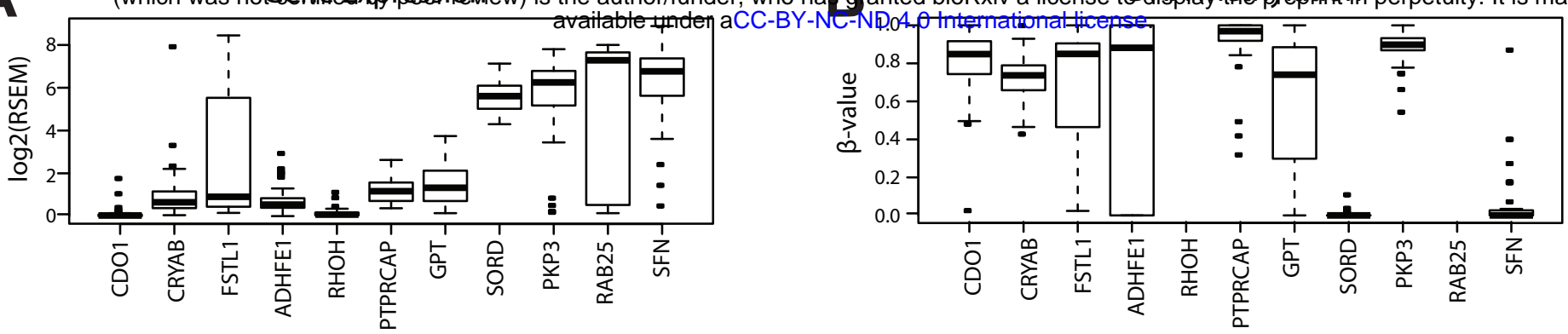

C

ADHFE1 in CCLE

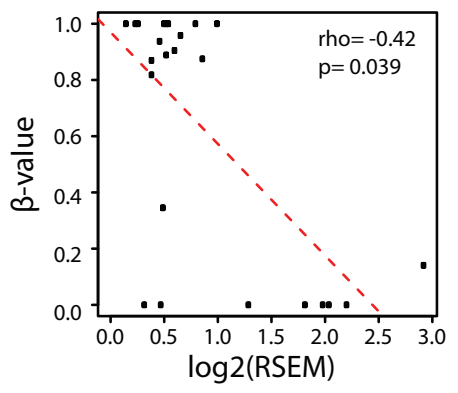

D

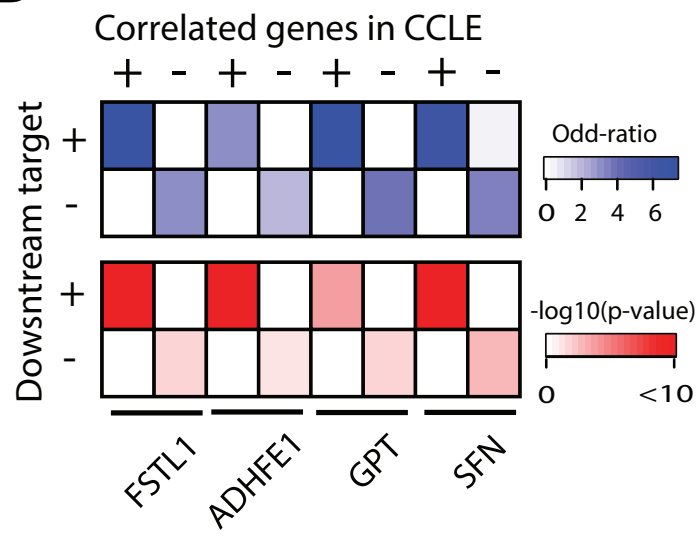

E

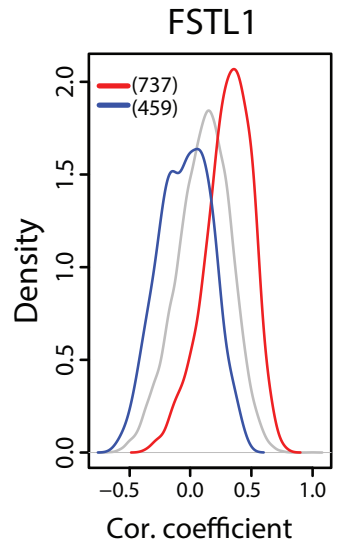

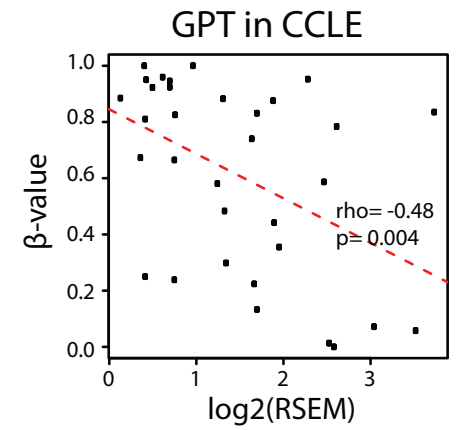
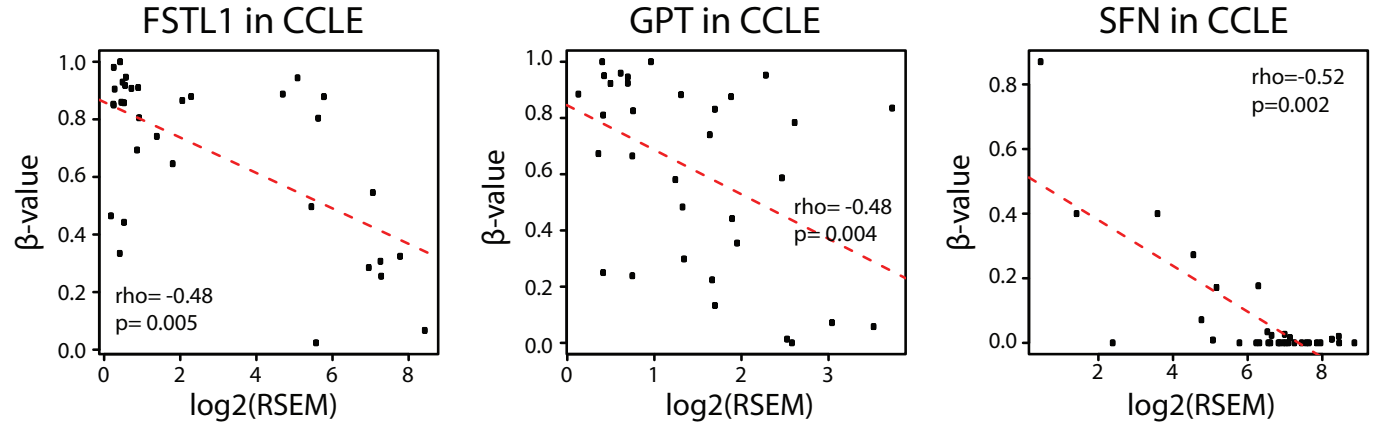

ADHFE1

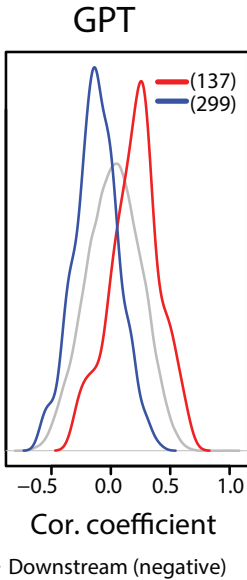

SFN
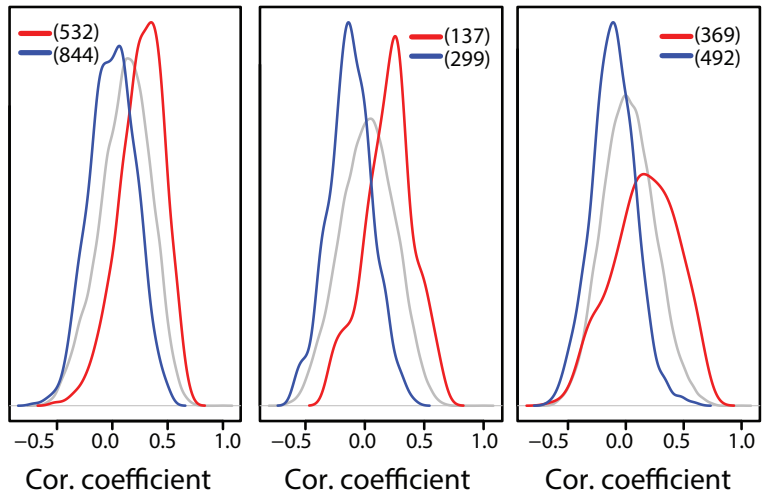

Downstream (positive)

Downstream (negative)

Background 


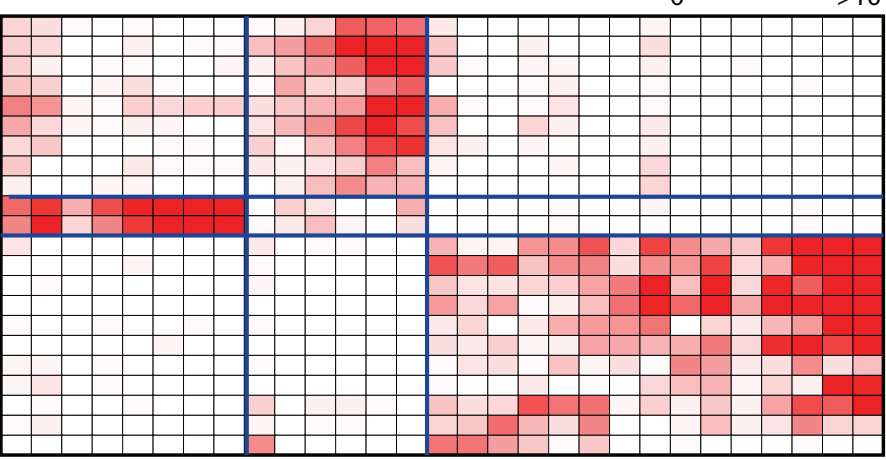

PKP3_neg

FSTL1_pos

SORD_neg

CDO1_pos

ADHFE1_pos

GPT neg

SFN_neg

$\mathrm{RHOH}$ pos

PTPRCAP_pos

PKP3 pos

FSTL1_neg

CDO1_neg

ADHFË1_neg

CRYAB_neg



PTPRCAP_neg

SFN_pos

RAB 25 pos

PTPRCAP_neg

SFN_pos

GPT_pos

-

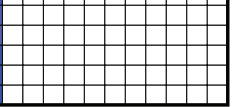

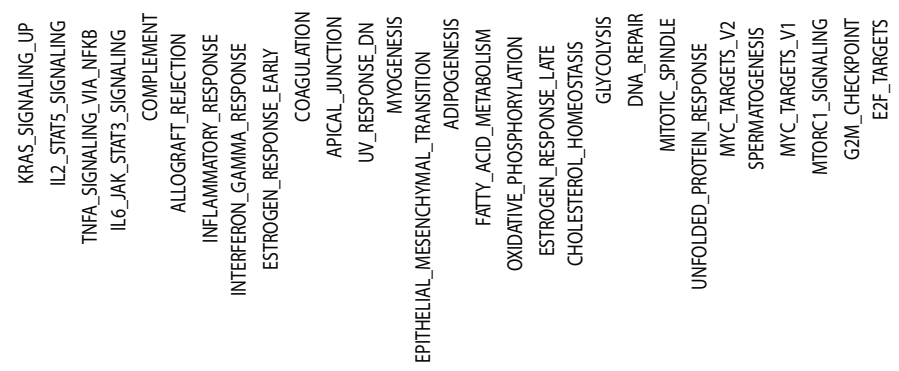




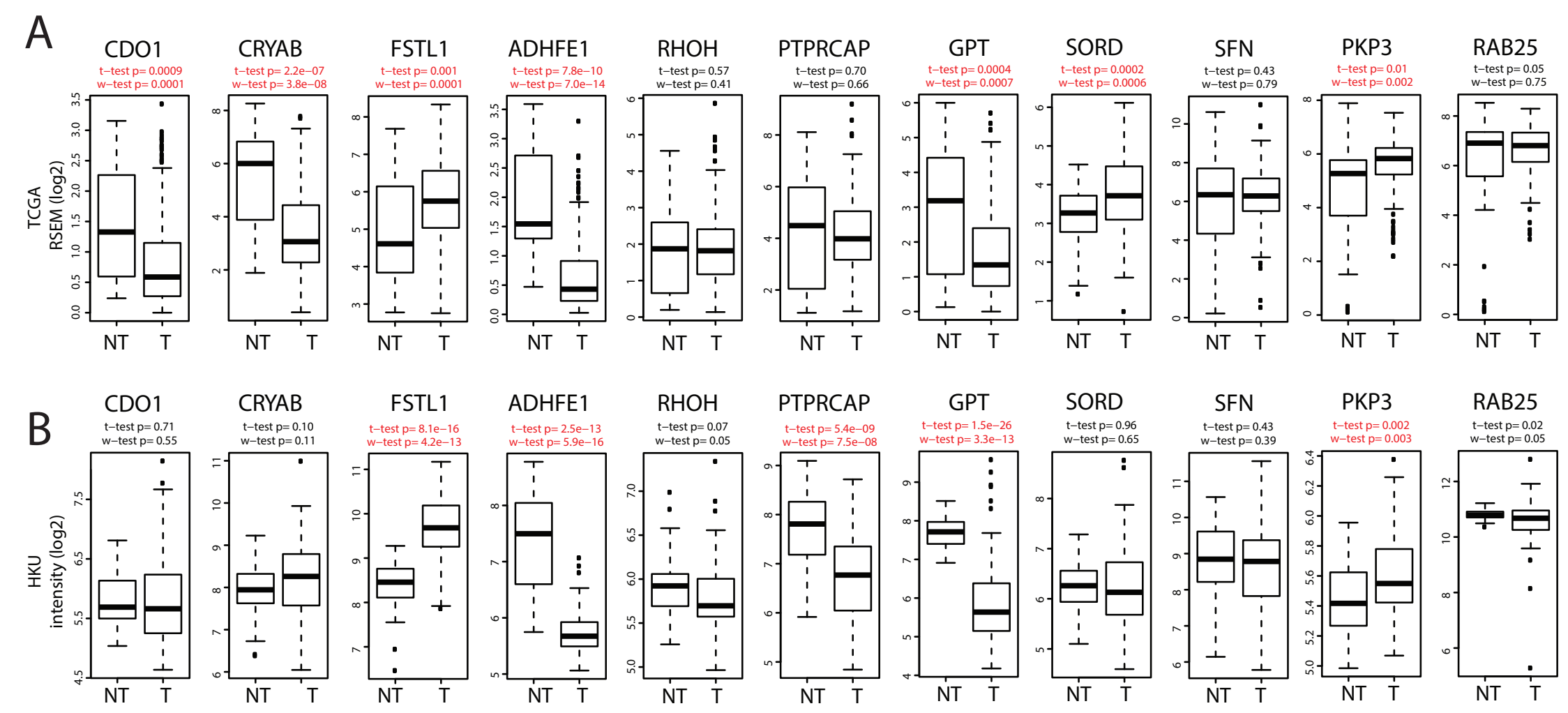



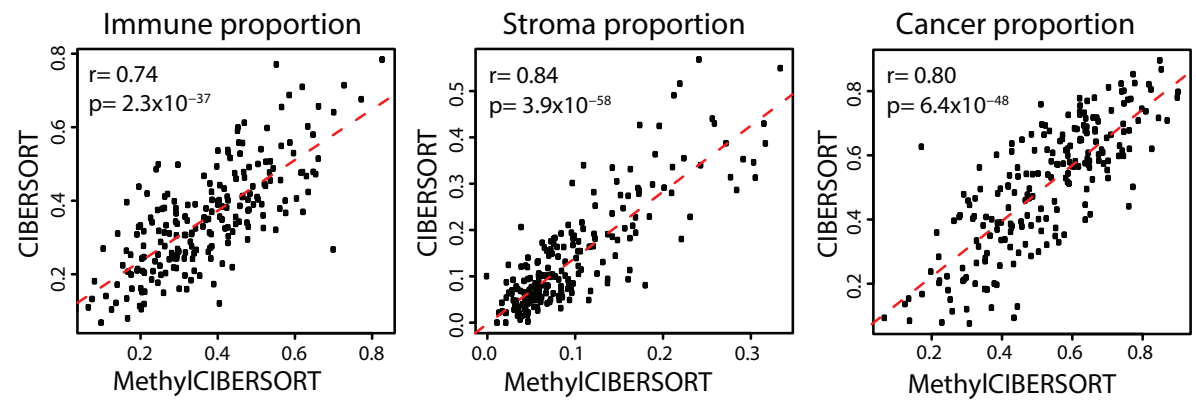